\title{
Some new methods in the Theory of $m$-Quasi-Invariants
}

\author{
J. Bell, A.M. Garsia and N. Wallach \\ Department of Mathematics \\ University of California, San Diego, USA \\ agarsia@math.ucsd.edu
}

Submitted: Jan 29, 2005; Accepted: Jul 15, 2005; Published: Aug 30, 2005

\begin{abstract}
We introduce here a new approach to the study of $m$-quasi-invariants. This approach consists in representing $m$-quasi-invariants as $N^{\text {tuples }}$ of invariants. Then conditions are sought which characterize such $N^{\text {tuples }}$. We study here the case of $S_{3} \mathrm{~m}$-quasi-invariants. This leads to an interesting free module of triplets of polynomials in the elementary symmetric functions $e_{1}, e_{2}, e_{3}$ which explains certain observed properties of $S_{3} \mathrm{~m}$-quasi-invariants. We also use basic results on finitely generated graded algebras to derive some general facts about regular sequences of $S_{n} m$-quasi-invariants
\end{abstract}

\section{Introduction}

The ring of polynomials in $x_{1}, x_{2}, \ldots, x_{n}$ with rational coefficients will be denoted $\mathbb{Q}\left[X_{n}\right]$. For $P \in \mathbb{Q}\left[X_{n}\right]$ we will write $P(x)$ for $P\left(x_{1}, x_{2}, \ldots, x_{n}\right)$.

Let us denote by $s_{i j}$ the transposition which interchanges $x_{i}$ with $x_{j}$. Note that for any pair $i, j$ and exponents $a, b$ we have the identities

$$
\frac{x_{i}^{a} x_{j}^{b}-x_{j}^{a} x_{i}^{b}}{x_{i}-x_{j}}= \begin{cases}x_{i}^{a} x_{j}^{a}\left(\sum_{r=0}^{b-a-1} x_{j}^{r} x_{i}^{b-a-1-r}\right) & \text { if } a \leq b, \\ x_{i}^{b} x_{j}^{b}\left(\sum_{r=0}^{a-b-1} x_{i}^{r} x_{j}^{a-b-1-r}\right) & \text { if } a>b .\end{cases}
$$

This shows that the ratio in (1.1) is always a polynomial that is symmetric in $x_{i}, x_{j}$. It immediately follows from (1.1) that the so-called "divided difference" operator

$$
\delta_{i j}=\frac{1}{x_{i}-x_{j}}\left(1-s_{i j}\right)
$$

sends polynomials into polynomials symmetric in $x_{i}, x_{j}$. 
It follows from this that for any $P \in \mathbb{Q}\left[X_{n}\right]$ the highest power of $\left(x_{i}-x_{j}\right)$ that divides the difference $\left(1-s_{i j}\right) P$ must necessarily be odd. This given, a polynomial $P \in \mathbb{Q}\left[X_{n}\right]$ is said to be " $m$-quasi-invariant" if and only if, for all pairs $1 \leq i<j \leq n$, the difference

$$
\left(1-s_{i j}\right) P(x)
$$

is divisible by $\left(x_{i}-x_{j}\right)^{2 m+1}$. The space of $m$-quasi-invariant polynomials in $x_{1}, x_{2}, \ldots, x_{n}$ will here and after be denoted " $\mathcal{Q} \mathcal{I}_{m}\left[X_{n}\right]$ " or briefly " $\mathcal{Q} \mathcal{I}_{m}$ ". Clearly $\mathcal{Q} \mathcal{I}_{m}$ is a vector space over $\mathbb{Q}$, moreover since the operators $\delta_{i j}$ satisfy the "Leibnitz" formula

$$
\delta_{i j} P Q=\left(\delta_{i j} P\right) Q+\left(s_{i j} P\right) \delta_{i j} Q
$$

we see that $\mathcal{Q} \mathcal{I}_{m}$ is also a ring. Note that we have the inclusions

$$
\begin{aligned}
\mathbb{Q}\left[X_{n}\right]= & \mathcal{Q \mathcal { I } _ { 0 }}\left[X_{n}\right] \supset \mathcal{Q I}_{1}\left[X_{n}\right] \supset \mathcal{Q I}_{2}\left[X_{n}\right] \supset \cdots \supset \mathcal{Q I}_{m}\left[X_{n}\right] \supset \cdots \supset \mathcal{Q} \mathcal{I}_{\infty}\left[X_{n}\right] \\
& =\mathcal{S Y M} \mathcal{M}\left[X_{n}\right] .
\end{aligned}
$$

where $\mathcal{S Y \mathcal { M }}\left[X_{n}\right]$ here denotes the ring of symmetric polynomials in $x_{1}, x_{2}, \ldots, x_{n}$.

It was recently shown by Etingof and Ginzburg [4] that each $\mathcal{Q I}_{m}\left[X_{n}\right]$ is a free module over $\mathcal{S Y \mathcal { M }}\left[X_{n}\right]$ of rank $n$ !. In fact, this is only the $S_{n}$ case of a general result that is proved in [4] for all Coxeter groups. There is an extensive literature (see [1], [3], [5], [7], [9]) covering several aspects of quasi-invariants. These spaces appear to possess a rich combinatorial underpinning resulting in truly surprising identities. The $S_{n}$ case deserves special attention since the results in this case extend in a remarkable manner many well known classical results that hold true for the familiar polynomial ring $\mathbb{Q}\left[X_{n}\right]$. To be precise note that for each $m$ we have the direct sum decomposition

$$
\mathcal{Q I}_{m}=\mathcal{H}_{0}\left[\mathcal{Q I}_{m}\right] \oplus \mathcal{H}_{1}\left[\mathcal{Q I}_{m}\right] \oplus \cdots \oplus \mathcal{H}_{k}\left[\mathcal{Q I}_{m}\right] \oplus \cdots
$$

where $\mathcal{H}_{k}\left[\mathcal{Q I}_{m}\right]$ denotes the subspace of $m$-quasi-invariants that are homogeneous of degree $k$. Since $m$-quasi-invariance and homogeneity are preserved by the $S_{n}$ action each $\mathcal{H}_{k}\left[\mathcal{Q} \mathcal{I}_{m}\right]$ is an $S_{n}$ module and we can thus define the graded Frobenius characteristic of $\mathcal{Q I}_{m}$ by setting

$$
\Phi_{m}(x ; q)=\sum_{k \geq 0} q^{k} F \operatorname{char} \mathcal{H}_{k}\left[\mathcal{Q \mathcal { I }}_{m}\right]
$$

where we denote by $F$ the Frobenius map. Now it is shown by Felder and Veselov in [6] that we have

$$
(1-q)\left(1-q^{2}\right) \cdots\left(1-q^{n}\right) \Phi_{m}(x ; q)=\sum_{\lambda \vdash n} S_{\lambda}\left(\sum_{T \in S T(\lambda)} q^{c o(T)}\right) q^{m\left(\left(\begin{array}{l}
n \\
2
\end{array}\right)-c_{\lambda}\right)}
$$

where $S_{\lambda}$ is the Schur function corresponding to $\lambda, S T(\lambda)$ denotes the collection of standard tableaux of shape $\lambda, \operatorname{co}(T)$ denotes the cocharge of $T$ and $c_{\lambda}$ gives the sum of the contents of the partition $\lambda$. This truly beautiful formula extends in a surprisingly simple manner the well known classical result for $m=0$. In fact, more is true. Since the ideal

$$
\left(e_{1}, e_{2}, \ldots, e_{n}\right)_{\mathcal{Q I}_{m}\left[X_{n}\right]}
$$


generated in $\mathcal{Q I}_{m}\left[X_{n}\right]$ by the elementary symmetric functions $e_{1}, e_{2}, \ldots, e_{n}$ is also $S_{n^{-}}$ invariant, it follows from the Etingov-Ginsburg result that the polynomial on the right hand side of (1.4) is none other than the graded Frobenius characteristic of the quotient

$$
\mathcal{Q I}_{m}\left[X_{n}\right] /\left(e_{1}, e_{2}, \ldots, e_{n}\right)_{\mathcal{Q I}_{m}\left[X_{n}\right]} .
$$

Unfortunately, the literature on quasi-invariants makes use of such formidable machinery that presently the theory is accessible only to a few. This given, the above examples should provide sufficient motivation for a further study of $S_{n} m$-quasi-invariants from a more elementary point of view.

In this vein we find particularly intriguing in (1.4) the degree shift of each isotypic component of $\mathcal{Q I}_{m}$ expressed by the presence of the factor

$$
q^{m\left(\left(\begin{array}{c}
n \\
2
\end{array}\right)-c_{\lambda}\right)} .
$$

This shift pops out almost magically from manipulations involving a certain KnizhnikZamolodchikov connection used in [6] to compute the graded character of $\mathcal{Q I}_{m}$.

The present work results from an effort to understand the underlining mechanism that produces this degree shift. In this paper we only deal with the $S_{3}$ case but the methods we introduce should provide a new approach to the general study of $m$-quasi-invariants.

The idea is to start with what is known when $m=0$ and determine the deformations that are needed to obtain $\mathcal{Q I}_{m}$. More precisely our point of departure is the following well known result.

Theorem 1.6 Every polynomial $P(x) \in \mathbf{Q}\left[X_{n}\right]$ has a unique expansion in the form

$$
P(x)=\sum_{x^{\epsilon} \in \mathcal{A} \mathcal{R} \mathcal{T}(n)} x^{\epsilon} A_{\epsilon}(x) \quad\left(\text { with } A_{\epsilon} \in \mathcal{S} \mathcal{Y} \mathcal{M}\left[X_{n}\right]\right)
$$

and

$$
\mathcal{A R T}(n)=\left\{x^{\epsilon}=x_{1}^{\epsilon_{1}} x_{1}^{\epsilon_{2}} \cdots x_{n}^{\epsilon_{n}}: 0 \leq \epsilon_{i} \leq i-1\right\},
$$

It follows from this that each $P(x) \in \mathbf{Q}\left[X_{n}\right]$ may be uniquely represented by a $n !^{\text {tuple }}$ of symmetric polynomials. The question then naturally arises as to what conditions these symmetric polynomials must satisfy so that $P(x)$ lies in $\mathcal{Q I}_{m}$. In this work we give a complete answer for $S_{3}$. Remarkably, we shall see that, even in this very special case, the answer stems from a variety of interesting developments. We should mention that Feigin and Veselov in [7] prove the freeness result of the $m$-quasi-invariants for all Dihedral groups. They do this by exhibiting a completely explicit basis for the quotients analogous to (1.5). Of course, since the $S_{3} m$-quasi-invariants are easily obtained from the $m$-quasiinvariants of the dihedral groupd $D_{3}$, in principle, the results in [7] should have a bearing on what we do here. However, as we shall see in the first section, the freeness result for $m$-quasi-invariants is quite immediate whenever the invariants form a polynomial ring on two generators. Moreover, the methods used in [7] are quite distinct from ours and don't reveal the origin of the observed degree shift. 
This paper is divided in to three sections. In the first section we start with a review of some basic facts and definitions concerning finitely generated graded algebras. Two noteworthy developments in this section are a very simple completely elementary proof of the freeness result for dihedral groups $m$-quasi-invariants and the remarkable fact that the freeness result for all $m$-quasi-invariants follows in a completely elementary manner from one single inequality. Namely that the quotient of the ring $m$-quasi-invariants by the ideal generated by the $G$-invariants has dimension bounded by the order of $G$. In the second section we determine the conditions that $6^{\text {tuples }}$ of symmetric functions give an element of $\mathcal{Q I}_{m}\left[X_{3}\right]$. It develops that the trivial and alternating representations are immediately dealt with. In the third section we show how that these conditions, for the 2-dimensional irreducible of $S_{3}$, lead to the construction of an interesting free module of triplets over the ring $\mathbb{Q}\left[e_{1}, e_{2}, e_{3}\right]$ which is at the root of the observed degree shift for $S_{3}$.

\section{Cohen-Macauliness and m-quasi-invariants.}

Before we can proceed with our arguments we need to introduce notation and state a few basic facts. To begin let us recall that the Hilbert series of a finitely generated, graded algebra $\mathcal{A}$ is given by the formal sum

$$
F_{\mathcal{A}}(t)=\sum_{m \geq 0} t^{m} \operatorname{dim} \mathcal{H}_{m}(\mathcal{A})
$$

where $\mathcal{H}_{m}(\mathcal{A})$ denotes the subspace spanned by the elements of $\mathcal{A}$ that are homogeneous of degree $m$. It is well known that $F_{\mathcal{A}}(t)$ is a rational function of the form

$$
F_{\mathcal{A}}(t)=\frac{P(t)}{(1-t)^{k}}
$$

with $P(t)$ a polynomial. The minimum $k$ for which this is possible characterizes the growth of $\operatorname{dim} \mathcal{H}_{m}(\mathcal{A})$ as $m \rightarrow \infty$. This integer is customarily called the "Krull dimension" of $\mathcal{A}$ and is denoted " $\operatorname{dim}_{K} \mathcal{A}$ ". It is easily shown that we can always find in $\mathcal{A}$ homogeneous elements $\theta_{1}, \theta_{2}, \ldots, \theta_{k}$ such that the quotient of $\mathcal{A}$ by the ideal generated by $\theta_{1}, \theta_{2}, \ldots, \theta_{k}$ is a finite dimensional vector space. In symbols

$$
\operatorname{dim} \mathcal{A} /\left(\theta_{1}, \theta_{2}, \ldots, \theta_{k}\right)_{\mathcal{A}}<\infty
$$

It is shown that $\operatorname{dim}_{K} \mathcal{A}$ is also equal to the minimum $k$ for which this is possible. When (2.2) holds true and $k=\operatorname{dim}_{K} \mathcal{A}$ then $\left\{\theta_{1}, \theta_{2}, \ldots, \theta_{k}\right\}$ is called a "homogeneous system of parameters", $\mathcal{H S O P}$ in brief.

It follows from $(2.2)$ that if $\eta_{1}, \eta_{2}, \ldots, \eta_{N}$ are a basis for the quotient in $(2.2)$ then every element of $\mathcal{A}$ has an expansion of the form

$$
P=\sum_{i=1}^{N} \eta_{i} P_{i}\left(\theta_{1}, \theta_{2}, \ldots, \theta_{k}\right)
$$


with coefficients $P_{i}\left(\theta_{1}, \theta_{2}, \ldots, \theta_{k}\right)$ polynomials in their arguments. The algebra $\mathcal{A}$ is said to be Cohen-Macaulay, when the coefficients $P_{i}\left(\theta_{1}, \theta_{2}, \ldots, \theta_{k}\right)$ are uniquely determined by $P$. This amounts to the requirement that the collection

$$
\left\{\eta_{i} \theta_{1}^{p_{1}} \theta_{2}^{p_{2}} \cdots \theta_{k}^{p_{k}}\right\}_{i, p}
$$

is a basis for $\mathcal{A}$ as a vector space. Note that when this happens and $\theta_{1}, \theta_{2}, \ldots, \theta_{k}$; $\eta_{1}, \eta_{2}, \ldots, \eta_{N}$ are homogeneous of degrees $d_{1}, d_{2}, \ldots, d_{k} ; r_{1}, r_{2}, \ldots, r_{N}$ then we must necessarily have

$$
F_{\mathcal{A}}(t)=\frac{\sum_{i=1}^{N} t^{r_{i}}}{\left(1-t^{d_{1}}\right)\left(1-t^{d_{2}}\right) \cdots\left(1-t^{d_{k}}\right)}
$$

from which it follows that $k=\operatorname{dim}_{K} \mathcal{A}$. It develops that this identity implies that, for any $i=1,2, \ldots, k$ the element $\theta_{i}$ is not a zero a zero divisor of the quotient

$$
\mathcal{A} /\left(\theta_{1}, \theta_{2}, \ldots, \theta_{i-1}\right)_{\mathcal{A}}
$$

We call such sequences $\theta_{1}, \theta_{2}, \ldots, \theta_{k}$ "regular". Conversely, if $\mathcal{A}$ has an $\mathcal{H S O P} \theta_{1}, \theta_{2}, \ldots, \theta_{k}$ that is a regular sequence, then (2.5) must hold true for any basis $\eta_{1}, \eta_{2}, \ldots, \eta_{N}$ of the quotient $\mathcal{A} /\left(\theta_{1}, \theta_{2}, \ldots, \theta_{k}\right)_{\mathcal{A}}$ and the uniqueness in the expansions (2.4) must necessarily follow yielding the Cohen-Macauliness of $\mathcal{A}$. However, for our applications to $m$-QuasiInvariants we need to make use of the following stronger criterion

Proposition 2.6 Let $\mathcal{A}$ be finitely generated graded algebra and $\theta_{1}, \theta_{2}, \ldots, \theta_{k}$ be an $\mathcal{H S O P}$ with $d_{i}=\operatorname{degree}\left(\theta_{i}\right)$, then $\mathcal{A}$ is Cohen-Macaulay and $\theta_{1}, \theta_{2}, \ldots, \theta_{k}$ is a regular sequence if and only if

$$
\lim _{t \rightarrow-1}\left(1-t^{d_{1}}\right)\left(1-t^{d_{2}}\right) \cdots\left(1-t^{d_{k}}\right) F_{\mathcal{A}}(t)=\operatorname{dim} \mathcal{A} /\left(\theta_{1}, \theta_{2}, \ldots, \theta_{k}\right)_{\mathcal{A}}
$$

This result is known. An elemtary proof of it may be found in [8].

A particular example which plays a role here is when $\mathcal{A}=\mathbb{Q}\left[x_{1}, x_{2}, \ldots, x_{n}\right]$ is the ordinary polynomial ring and the $\mathcal{H S O P}$ is the sequence $e_{1}, e_{2}, \ldots, e_{n}$ of elementary symmetric functions. As we mentioned in the introduction following result is well known but for sake of completeness we give a sketch of the proof.

Theorem 2.8 Every polynomial $P(x) \in \mathbb{Q}\left[x_{1}, x_{2}, \ldots, x_{n}\right]$ has a unique expansion of the form

$$
P(x)=\sum_{x^{\epsilon} \in \mathcal{A R T}(n)} x^{\epsilon} P_{\epsilon}\left(e_{1}, e_{2}, \ldots, e_{n}\right)
$$

where

$$
\mathcal{A R T}(n)=\left\{x^{\epsilon}=x_{1}^{\epsilon_{1}} x_{2}^{\epsilon_{2}} \cdots x_{n}^{\epsilon_{n}}: 0 \leq \epsilon_{i} \leq i-1\right\}
$$

In particular $e_{1}, e_{2}, \ldots, e_{n}$ is a regular sequence. 
Proof It is easily seen that we have

$$
\prod_{i=1}^{n} \frac{1}{1-t x_{i}} \cong 1
$$

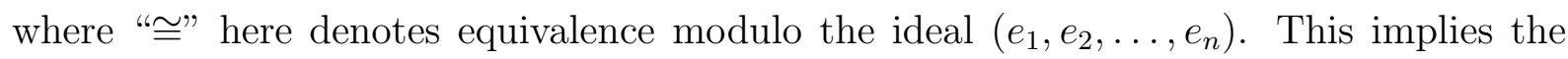
identity

$$
\prod_{j=1}^{i-1}\left(1-t x_{j}\right) \cong \prod_{j=i}^{n} \frac{1}{\left(1-t x_{j}\right)}=\sum_{r \geq 0} h_{r}\left(x_{i}, x_{i+1} \ldots, x_{n}\right) t^{r} .
$$

Equating coefficients of $t^{i}$ we derive that

$$
0 \cong h_{i}\left(x_{i}, x_{i+1} \ldots, x_{n}\right)
$$

Now this gives

$$
x_{i}^{i} \cong \sum_{j=0}^{i-1} x_{i}^{j} h_{i-j}\left(x_{i+1} \ldots, x_{n}\right) \quad(\text { for } 1 \leq i \leq n-1)
$$

as well as

$$
x_{n}^{n} \cong 0 \text {. }
$$

It is easily seen that (2.10) and (2.11) yield an algorithm for expressing, modulo the ideal $\left(e_{1}, e_{2}, \ldots, e_{n}\right)$, every monomial as a linear combination of monomials in $\mathcal{A R \mathcal { T }}(n)$. This implies that the collection

$$
\left\{x^{\epsilon} e_{1}^{p_{1}} e_{2}^{p_{2}} \cdots e_{n}^{p_{n}}: x^{\epsilon} \in \mathcal{A} \mathcal{R} \mathcal{T}(n) ; p_{i} \geq 0\right\}
$$

spans $\mathbb{Q}\left[x_{1}, x_{2}, \ldots, x_{n}\right]$. In particular we derive the coefficient-wise inequality

$$
F_{\mathbb{Q}\left[x_{1}, x_{2}, \ldots, x_{n}\right]}(t)<<\frac{\prod_{i=2}^{n}\left(1+t+\cdots+t^{i-1}\right)}{(1-t)\left(1-t^{2}\right) \cdots\left(1-t^{n}\right)}=\frac{1}{(1-t)^{n}}
$$

since

$$
F_{\mathbb{Q}\left[x_{1}, x_{2}, \ldots, x_{n}\right]}(t)=\frac{1}{(1-t)^{n}}
$$

equality must hold in (2.13), but that implies that the collection in (2.12) has the correct number of elements in each degree and must therefore be a basis, proving uniqueness for the expansions in (2.18).

We can now apply these observations to the study of $m$-quasi-invariants. To begin note that, we have the following useful fact

Theorem 2.14 To prove that $e_{1}, e_{2}, \ldots, e_{n}$ is a regular sequence in $\mathcal{Q I}_{m}\left[X_{n}\right]$ we need only construct a spanning set of $n$ ! elements for the quotient

$$
\mathcal{Q I}_{m}\left[X_{n}\right] /\left(e_{1}, e_{2}, \ldots, e_{n}\right)_{\mathcal{Q} \mathcal{I}_{m}\left[X_{n}\right]}
$$

In particular the Cohen-Macauliness of $\mathcal{Q I}_{m}\left[X_{n}\right]$ is equivalent to the statement that this quotient has $n$ ! dimensions. 
Proof Let $\Pi(x)$ denote the Vandermonde determinant

$$
\Pi(x)=\prod_{1 \leq i<j \leq n}\left(x_{i}-x_{j}\right) .
$$

This given, it is easy to see that the map

$$
P(x) \longrightarrow \Pi(x)^{2 m} P(x)
$$

is an injection of $\mathbb{Q}\left[x_{1}, x_{2}, \ldots, x_{n}\right]$ into $\mathcal{Q} \mathcal{I}_{m}\left[X_{n}\right]$. This fact combined with the inclusion $\mathcal{Q I}_{m}\left[X_{n}\right] \subseteq \mathbb{Q}\left[x_{1}, x_{2}, \ldots, x_{n}\right]$ yields the coefficient-wise Hilbert series inequalities

$$
\frac{t^{n(n-1) m}}{(1-t)^{n}}<<F_{\mathcal{Q I}_{m}\left[X_{n}\right]}(t)<<\frac{1}{(1-t)^{n}}
$$

this gives

$$
\lim _{t \rightarrow-1}(1-t)\left(1-t^{2}\right) \cdots\left(1-t^{n}\right) F_{\mathcal{Q} \mathcal{I}_{m}\left[X_{n}\right]}(t)=n ! .
$$

Thus if $e_{1}, e_{2}, \ldots, e_{n}$ is a regular in sequence in $\mathcal{Q I}_{m}\left[X_{n}\right],(2.25)$ then the quotient

$$
\mathcal{Q I}_{m}\left[X_{n}\right] /\left(e_{1}, e_{2}, \ldots, e_{n}\right)_{\mathcal{Q} \mathcal{I}_{m}\left[X_{n}\right]}
$$

must be of dimension $n$ !. To prove the converse, note that if we have a homogeneous basis $\eta_{1}, \eta_{2}, \ldots \eta_{N}$,of degrees $r_{1}, r_{2}, \ldots, r_{n}$, for this quotient, then we the Hilbert series inequality

$$
F_{\mathcal{Q I}_{m}\left[X_{n}\right]}(t)<<\frac{\sum_{i=1}^{N} t^{r_{i}}}{(1-t)\left(1-t^{2}\right) \cdots\left(1-t^{n}\right)}
$$

combined with (2.16) yields that

$$
n ! \leq N .
$$

On the other hand if we have a spanning set of $n$ ! elements for the quotient in (2.17) we must also have

$$
N \leq n !
$$

This forces the equality

$$
\lim _{t \rightarrow-1}(1-t)\left(1-t^{2}\right) \cdots\left(1-t^{n}\right) F_{\mathcal{Q I}_{m}\left[X_{n}\right]}(t)=\operatorname{dim} \mathcal{Q I}_{m}\left[X_{n}\right] /\left(e_{1}, e_{2}, \ldots, e_{n}\right)_{\mathcal{Q I}_{m}\left[X_{n}\right]} .
$$

Thus we can apply Proposition 2.6 and derive that $e_{1}, e_{2}, \ldots, e_{n}$ is a regular sequence in $\mathcal{Q I}_{m}\left[X_{n}\right]$. This completes our argument.

It develops that the regularity of $e_{1}, e_{2}, e_{3}$, can be shown in a very elementary fashion for all $n$. This of course implies the Cohen-Macauliness of $\mathcal{Q I}\left[X_{3}\right]$. But before we give the general argument it will be good to go over the case of $e_{1}, e_{2}, e_{3}$ in $\mathcal{Q I}\left[X_{3}\right]$. In fact, we can proceed a bit more generally and work in the Dihedral group setting.

Let us recall that the Dihedral group $D_{n}$ is the group of transformations of the $x, y$ plane generated by the reflection $T$ across the $x$-axis and a rotation $R_{n}$ by $2 \pi / n$. In complex notation we may write

$$
T z=\bar{z}, \quad \text { and } \quad R_{n} z=e^{2 \pi i / n} z
$$


It follows from this that the two fundamental invariants of $D_{n}$ are

$$
p_{2}=x^{2}+y^{2}, \quad \text { and } \quad g_{n}=\operatorname{Re} z^{n}=\sum_{r=0}^{\lfloor n / 2\rfloor}\left(\begin{array}{c}
n \\
2 r
\end{array}\right)(-1)^{r} x^{n-2 r} y^{2 r} .
$$

Note that if $n=2 k$ and we set

$$
P(t)=\sum_{r=0}^{k}\left(\begin{array}{l}
2 k \\
2 r
\end{array}\right)(-1)^{r} t^{k-r}
$$

then we may write

$$
P(t)=P(-1)+(1+t) Q(t)
$$

with $Q(t)$ a polynomial of degree $k-1$. Now setting $t=x^{2} / y^{2}$ in (2.20) and mutiplying both sides by $y^{2 k}$ we get, since $P(-1)=(-1)^{k} 2^{2 k-1}$

$$
g_{n}(x, y)=(-1)^{k} 2^{2 k-1} y^{2 k}+p_{2}(x, y) y^{2 k-2} Q\left(x^{2} / y^{2}\right) \text {. }
$$

This shows that $y^{2 k}$ lies in the ideal $\left(p_{2}, g_{n}\right)_{\mathbb{Q}[x, y]}$. In particular, under the total order $x>y$ we derive that $x^{2}$ and $y^{2 k}$ lie in the upper set of leading monomials of the elements of this ideal. It follows that the monomials

$$
1, y, y^{2}, \ldots, y^{2 k-1} ; x, x y, x y^{2}, \ldots, x y^{2 k-1}
$$

span the quotient

$$
\mathbb{Q}[x, y] /\left(p_{2}, g_{n}\right) \mathbb{Q}[x, y]
$$

This forces the Hilbert series inequality

$$
F_{\mathbb{Q}[x, y]}(t)<<\frac{(1+t)\left(1+t+\cdots+t^{2 k-1}\right)}{\left(1-t^{2}\right)\left(1-t^{2 k}\right)}=\frac{1}{(1-t)^{2}}
$$

since we also have

$$
F_{\mathbb{Q}[x, y]}(t)=\frac{1}{(1-t)^{2}}
$$

It follows that the monomials in (2.21) are in fact a basis for the quotient in (2.22). An analogous argument yields a similar result when $n=2 k+1$. We need only observe that in this case we use the polynomial

$$
P(t)=\sum_{r=0}^{k}\left(\begin{array}{c}
2 k+1 \\
2 r
\end{array}\right)(-1)^{r} t^{r}
$$

and the total order $y>x$ to obtain that $y^{2}$ and $x^{2 k+1}$ are in the upper set of leading monomials of the ideal $\left(p_{2}, g_{n}\right)_{\mathbb{Q}[x, y]}$. This implies that

$$
1, x, x^{2}, \ldots, x^{2 k} ; y, y x, y x^{2}, \ldots, y x^{2 k}
$$

are a basis of the quotient in (2.22). Thus in either case we obtain that and that $p_{2}, g_{n}$ are a regular sequence in $\mathbb{Q}[x, y]$.

It develops that this immediately implies the Cohen Macauliness the $\operatorname{ring} \mathcal{Q} \mathcal{I}_{m}\left(D_{n}\right)$ of $m$-quasi-invariants of $D_{n}$. More precisely we have 
Theorem 2.23 The $D_{n}$ invariants $p_{2}, g_{n}$ are a regular sequence in $\mathcal{Q} \mathcal{I}_{m}\left(D_{n}\right)$.

Proof By definition, a polynomial $P(x, y) \in \mathbb{Q}[x, y]$ is said to be $D_{n} m$-quasi-invariant if and only if for any reflection $s$ of $D_{n}$ we have

$$
(1-s) P(x, y)=\alpha_{s}(x, y)^{2 m+1} P^{\prime}(x, y) \quad\left(P^{\prime}(x, y) \in \mathbb{Q}[x, y]\right)
$$

where $\alpha_{s}(x, y)$ denotes the equation of the line accross which $s$ reflects. This given, since $\mathcal{Q I}_{m}\left(D_{n}\right) \subseteq \mathbb{Q}[x, y]$, we clearly see that $p_{2}$ itself is not a zero divisor in $\mathcal{Q I}_{m}\left(D_{n}\right)$. So we need only show that $g_{n}$ is not a zero divisor modulo $\left(p_{2}\right)_{\mathcal{Q} \mathcal{I}_{m}\left(D_{n}\right)}$. Now suppose that for some $H \in \mathcal{Q I}_{m}\left(D_{n}\right)$ we have

$$
H g_{n}=p_{2} K \quad\left(\text { with } K \in \mathcal{Q I}_{m}\left(D_{n}\right)\right) \text {. }
$$

Then since $p_{2}, g_{n}$ are regular in $\mathbb{Q}[x, y]$ it follows that for some $K^{\prime} \in \mathbb{Q}[x, y]$ we have

$$
H=p_{2} K^{\prime}
$$

applying $1-s$ to both sides the invariance of $p_{2}$ gives

$$
(1-s) H(x, y)=\left(x^{2}+y^{2}\right)(1-s) K^{\prime}(x, y)
$$

and the $m$-quasi-invariance of $H$ yields that $\alpha_{s}(x, y)^{2 m+1}$ divides the right hand side. Since $x^{2}+y^{2}$ has no real factor, the polynomial $(1-s) K^{\prime}(x, y)$ must be divisible by $(x, y)^{2 m+1}$. This shows that $K^{\prime} \in \mathcal{Q I}_{m}\left(D_{n}\right)$ proving that $g_{n}$ in not a zero divisor in $\left(p_{2}\right)_{\mathcal{Q} \mathcal{I}_{m}\left(D_{n}\right)}$ and our argument is complete.

Our next step is to use the fact that the Weyl group of $A_{2}$ is $D_{3}$ to derive the CohenMacauliness of $\mathcal{Q} \mathcal{I}_{m}\left[X_{3}\right]$. To this end set

$$
f_{1}=(1,0,0), \quad f_{2}=(0,1,0), \quad f_{1}=(0,0,1) .
$$

and take as basis for the plane

$$
\Pi=\left\{\left(x_{1}, x_{2}, x_{3}\right): x_{1}+x_{2}+x_{3}=0\right\}
$$

the orthonormal vectors

$$
u=\sqrt{\frac{2}{3}}\left(\frac{f_{1}+f_{2}}{2}-f_{3}\right), \quad v=\frac{1}{\sqrt{2}}\left(f_{2}-f_{1}\right) .
$$

This gives the expansions

$$
\frac{1}{\sqrt{2}}\left(f_{1}-f_{2}\right)=-v, \quad \frac{1}{\sqrt{2}}\left(f_{1}-f_{3}\right)=\frac{\sqrt{3}}{2} u-\frac{1}{2} v, \quad \frac{1}{\sqrt{2}}\left(f_{2}-f_{3}\right)=\frac{\sqrt{3}}{2} u+\frac{1}{2} v .
$$

Note that we also have

$$
x u+y v=f_{1}\left(\frac{1}{\sqrt{6}} x-\frac{1}{\sqrt{2}} y\right)+f_{2}\left(\frac{1}{\sqrt{6}} x+\frac{1}{\sqrt{2}} y\right)-f_{3} \sqrt{\frac{2}{3}} x
$$


Since the vector

$$
\left(x_{1}-e_{1} / 3 x_{2}-e_{1} / 3 \quad x_{3}-e_{1} / 3\right) \quad\left(\text { with } e_{1}=x_{1}+x_{2}+x_{3}\right)
$$

lies in the plane $\Pi$ we can find $x, y$ giving

$$
x_{1}-e_{1} / 3=\frac{1}{\sqrt{6}} x-\frac{1}{\sqrt{2}} y, \quad x_{2}-e_{1} / 3=\frac{1}{\sqrt{6}} x+\frac{1}{\sqrt{2}} y, \quad x_{3}-e_{1} / 3=-\sqrt{\frac{2}{3}} x
$$

Solving these equations for $x$ and $y$ gives

$$
x=\frac{1}{\sqrt{6}}\left(e_{1}-3 x_{3}\right), \quad y=\frac{1}{\sqrt{2}}\left(x_{2}-x_{1}\right)
$$

Thus the substitution maps

$$
\phi: \mathbb{Q}\left[x_{1}, x_{2}, x_{3}\right] \longrightarrow \mathbb{Q}[x, y], \quad \psi: \mathbb{Q}[x, y] \longrightarrow \mathbb{Q}\left[x_{1}, x_{2}, x_{3}\right]
$$

defined by setting

$$
\phi P\left(x_{1}, x_{2}, x_{3}\right)=P\left(\phi\left(x_{1}\right), \phi\left(x_{2}\right), \phi\left(x_{3}\right)\right), \quad \psi Q(x, y)=Q(\psi(x), \psi(y))
$$

with

$$
\phi\left(x_{1}\right)=\frac{1}{\sqrt{6}} x-\frac{1}{\sqrt{2}} y, \quad \phi\left(x_{2}\right)=\frac{1}{\sqrt{6}} x+\frac{1}{\sqrt{2}} y, \quad \phi\left(x_{3}\right)=-\sqrt{\frac{2}{3}} x
$$

and

$$
\psi(x)=\frac{1}{\sqrt{6}}\left(e_{1}-3 x_{3}\right), \quad \psi(y)=\frac{1}{\sqrt{2}}\left(x_{2}-x_{1}\right)
$$

satisfy the identities

$$
x_{1}=\psi \phi\left(x_{1}\right)+e_{1} / 3, \quad x_{1}=\psi \phi\left(x_{2}\right)+e_{1} / 3, \quad x_{1}=\psi \phi\left(x_{3}\right)+e_{1} / 3 .
$$

In particular it follows that for $P\left(x_{1}, x_{2}, x_{3}\right) \in \mathbb{Q}\left[x_{1}, x_{2}, x_{3}\right]$ we will have

$$
P\left(x_{1}, x_{2}, x_{3}\right)=\psi \phi P\left(x_{1}, x_{2}, x_{3}\right)+e_{1} Q\left(x_{1}, x_{2}, x_{3}\right)
$$

with $Q\left(x_{1}, x_{2}, x_{3}\right) \in \mathbb{Q}\left[x_{1}, x_{2}, x_{3}\right]$. Moreover, a simple calculation with the elementary symmetric functions

$$
e_{1}=x_{1}+x_{2}+x_{3}, \quad e_{2}=x_{1} x_{2}+x_{1} x_{3}+x_{2} x_{3}, \quad e_{3}=x_{1} x_{2} x_{3}
$$

gives

$$
\phi\left(e_{1}\right)=0, \quad \phi\left(e_{2}\right)=-\frac{x^{2}+y^{2}}{2}, \quad \phi\left(e_{3}\right)=\frac{1}{3 \sqrt{6}}\left(x^{3}-3 x y^{2}\right)=\frac{1}{3 \sqrt{6}} g_{3}(x, y) .
$$

We have now all the ingredients needed to prove 
Theorem 2.28 The elementary symmetric functions $e_{1}, e_{2}, e_{3}$ are a regular sequence in $\mathcal{Q I}_{m}\left[X_{3}\right]$.

Proof Clearly, $e_{1}$ is not a zero divisor in $\mathcal{Q I}_{m}\left[X_{3}\right]$. Likewise, we can show that $e_{2}$ is not a zero divisor in $\mathcal{Q I}_{m}\left[X_{3}\right] /\left(e_{1}\right)_{\mathcal{Q I}_{m}\left[X_{3}\right]}$ in exactly the same way we showed that $g_{n}$ is not a zero divisor in $\mathcal{Q I}_{m}\left(D_{n}\right) /\left(p_{2}\right)_{\mathcal{Q} \mathcal{I}_{m}\left(D_{n}\right)}$. The only remaining step is to show that

$$
h e_{3}=A e_{1}+B e_{2} \quad \text { with } \quad h, A, B \in \mathcal{Q I}_{m}\left[X_{3}\right]
$$

implies

$$
h=\bar{A} e_{1}+\bar{B} e_{2} \quad \text { with } \quad \bar{A}, \bar{B} \in \mathcal{Q} \mathcal{I}_{m}\left[X_{3}\right] .
$$

Note that using the relations in (2.27), (2.29) gives

$$
\phi(h) \frac{1}{3 \sqrt{6}}\left(x^{3}-3 x y^{2}\right)=-\phi(B)\left(x^{2}+y^{2}\right) / 2
$$

Since $\phi$ maps $S_{3} m$-quasi-invariants onto $D_{3} m$-quasi-invariants, from Theorem 2.23 we derive that

$$
\phi(h)=C(x, y)\left(x^{2}+y^{2}\right)
$$

with $C(x, y)$ a $D_{3} m$-quasi-invariant. Applying $\psi$ to both sides and using (2.38) we get

$$
h=\psi(C) \psi\left(\left(x^{2}+y^{2}\right)\right)+e_{1} D
$$

with a suitable polynomial $D$. But

$$
\begin{aligned}
\psi\left(x^{2}+y^{2}\right) & =\frac{1}{6}\left(e_{1}^{2}-6 e_{1} x_{3}+9 x_{3}^{2}\right)+\frac{1}{2}\left(x_{2}^{2}+x_{1}^{2}-2 x_{1} x_{2}\right) \\
& =\frac{1}{6}\left(e_{1}^{2}-6 e_{1} x_{3}+9 x_{3}^{2}+3 x_{2}^{2}+3 x_{1}^{2}-6 x_{1} x_{2}\right) \\
& =\frac{1}{6}\left(e_{1}^{2}-6\left(x_{1} x_{3}+x_{2} x_{3}+x_{3}^{2}\right)+9 x_{3}^{2}+3 x_{2}^{2}+3 x_{1}^{2}-6 x_{1} x_{2}\right) \\
& =\frac{1}{6}\left(4 e_{1}^{2}-12 e_{2}\right)=\frac{2}{3} e_{1}^{2}-2 e_{2}
\end{aligned}
$$

Thus combining (2.32) and (2.33) we obtain

$$
h=\psi(C)\left(\frac{2}{3} e_{1}^{2}-2 e_{2}\right)+e_{1} D
$$

Since $\psi(C)$ is an $S_{3} m$-quasi-invariant and $\frac{2}{3} e_{1}^{2}-2 e_{2}$ is invariant, this relation forces $D$ to be $S_{3} m$-quasi-invariant as well and our argument is complete.

We terminate this section by showing that the mechanism we have used for passing from the Weyl group of $A_{2}$ to $S_{3}$ can be extended to all $n$. More precisely we can show that

Theorem 2.35 For any $1<i_{2}<i_{3} \leq n$ the elementary symmetric functions $e_{1}, e_{i_{2}}, e_{i_{3}}$ are a regular sequence in $\mathcal{Q} \mathcal{I}_{m}\left[X_{n}\right]$. 


\section{Proof}

We start by noting that the same argument we used for $D_{n}$ yields that for any $1<$ $i_{2} \leq n$ the two elementary symmetric functions $e_{1}, e_{i_{2}}$ are a regular sequence in $\mathcal{Q I}_{m}\left[X_{n}\right]$. So the extension of the previous argument consists in deriving from this that $e_{i_{3}}$ is not a zero divisor in $\mathcal{Q I}_{m}\left(X_{n}\right] /\left(e_{1}, e_{i_{2}}\right)_{\mathcal{Q} \mathcal{I}_{m}\left[X_{n}\right]}$. Since $e_{i_{2}}, e_{i_{3}}$ are basic $S_{n}$-invariants for the polynomials on the space $V=\left\{x_{1}+x_{2}+\cdots+x_{n}=0\right\}$, this particular step is a consequence of the following general result. To state it we need some definitions.

Let $\lambda(x)=a_{1} x_{1}+\cdots+a_{n} x_{n}$ be a nonzero homogeneous polynomial in $n$ variables and let $u$ be such that $\lambda(u)=1$. Let $R$ be a subalgebra of the algebra of polynomials on $V$. If $f$ is a polynomial on $V$ we extend $f$ to $\mathbb{Q}^{n}$ by setting $f(v+t u)=f(v)$. If $g \in \mathbb{Q}\left[x_{1}, \ldots, x_{n}\right]$ then we write $\bar{g}$ for the restiction $g_{\mid V}$ of $g$ to $V$. Let $S$ be the subalgebra of $\mathbb{Q}\left[x_{1}, x_{2}, \ldots, x_{n}\right]$ generated by the extensions of the elements of $R$ and $\lambda$. This given we have

Theorem 2.36 If $f_{1}, \ldots, f_{k}$ is a regular sequence in $R$ then $\lambda, f_{1}, \ldots, f_{k}$ is a regular sequence in $S$.

\section{Proof}

Every element, $f$, of $S$ has a unique expansion (ignoring coefficients that are 0)

$$
f=\bar{f}+f_{1} \lambda+\cdots+f_{d} \lambda^{d}
$$

with $f_{i} \in R$. Clearly $\lambda$ is not a zero divisor in $S$. Suppose that $g \in S$ and $g f_{1} \in S \lambda$. Then

$$
g=\bar{g}+g_{1} \lambda+\cdots+g_{r} \lambda^{r}
$$

with $\bar{g}, g_{1}, \ldots, g_{r} \in R$. Restricting to $V$ we have $\bar{g} f_{1}=0$. Since $f_{1}$ is not a zero divisor in $R$ this implies that $\bar{g}=0$. Hence $g=\lambda\left(g_{1}+\cdots+g_{r} \lambda^{r-1}\right)=\lambda h$ with $h \in S$. Assume that we have shown that $\lambda, f_{1}, \ldots, f_{j-1}$ is a regular sequence in $S$. Suppose that we have

$$
g f_{j}=h_{0} \lambda+h_{1} f_{1}+\cdots+h_{j-1} f_{j-1}
$$

with $h_{l} \in S$ for $l=0, \ldots, j-1$. Restricting both sides of this equation to $V$ we get

$$
\bar{g} f_{j}=\overline{h_{1}} f_{1}+\cdots+\overline{h_{j-1}} f_{j-1} \text {. }
$$

Here $\overline{h_{l}} \in R$ for $l=1, \ldots, j-1$ and since $f_{1}, \ldots, f_{j}$ is a regular sequence in $R$ this implies that

$$
\bar{g}=\gamma_{1} f_{1}+\cdots+\gamma_{j-1} f_{j-1} \quad\left(\text { with } \gamma_{i} \in R \text { for } i=1, \ldots, j-1 .\right)
$$

Now $g=\bar{g}+g_{1} \lambda+\cdots+g_{r} \lambda^{r}$ with $g_{i} \in R$. Thus $g-\bar{g}=\lambda\left(g_{1}+\cdots+g_{r} \lambda^{r-1}\right)=\lambda h$ with $h \in S$. Hence

$$
g=\gamma_{1} f_{1}+\cdots+\gamma_{j-1} f_{j-1}+\lambda h .
$$

This completes the proof.

To apply this result to $m$-quasi-invariants. We take $\lambda(x)=e_{1}=x_{1}+\cdots+x_{n}$, $u=(1, \ldots, 1) / n$ and $V$ the zero set of $e_{1}$. Finally we take $R$ be the $S_{n} m$-quasi-invariants polynomials on $V$ and let $S=\mathcal{Q I}_{M}\left[X_{n}\right]$. The only missing ingredient is given by the following 
Lemma 2.37 $\mathcal{Q} \mathcal{I}_{m}\left[X_{n}\right]$ is the subalgebra of $\mathbb{Q}\left[x_{1}, \ldots, x_{n}\right]$ generated by $R$ and $e_{1}$.

Proof First observe that if $g=h e_{1}$ and $g \in \mathcal{Q I}_{m}\left[X_{n}\right]$ then $h \in \mathcal{Q I}_{m}\left[X_{n}\right]$. Indeed, if $\alpha$ is a root of $A_{n-1}$ then $\left(1-s_{\alpha}\right) g=\left(\left(1-s_{\alpha}\right) h\right) e_{1}$. Now $\left(1-s_{\alpha}\right) g=\alpha^{2 m+1} w$. Thus have

$$
\alpha^{2 m+1} w=\left(\left(1-s_{\alpha}\right) h\right) e_{1} .
$$

But $\alpha$ and $e_{1}$ are relatively prime. Hence $e_{1}$ divides $w$. That is $w=\phi e_{1}$. Hence $\left(\left(1-s_{\alpha}\right) h\right) e_{1}=\alpha^{2 m+1} \phi e_{1}$. Dividing off $e_{1}$ yields the $m$-quasi-invariance of $h$.

We need to show that if $f \in \mathcal{Q I}_{m}\left[X_{n}\right]$ and if

$$
f=f_{0}+f_{1} e_{1}+\cdots+f_{r} e_{1}^{r}
$$

with $f_{i}$ polynomials on $V$ then $f_{i} \in R$ for all $i$. Note that the assertion is trivially true for $r=0$. We can thus proceed by induction on $r$ and assume the assertion true up to $r-1$. To prove it for $r$ note that if we restrict both sides of (2.38) to $V$ we have $\bar{f}=f_{0}$. Since $f \in \mathcal{Q I}_{m}\left[X_{n}\right], \bar{f} \in R$. Thus $f_{0} \in R$. Now $f-f_{0}=e_{1}\left(f_{1}+\cdots+f_{r} e_{1}^{r-1}\right)$. From the observation at the beginning of the proof we derive that $f_{1}+\cdots+f_{r} e_{1}^{r-1} \in \mathcal{Q I}_{m}\left[X_{3}\right]$ and the induction hypothesis completes the argument.

\section{More on $\mathbf{S}_{3}$ m-quasi-invariants.}

Using Theorem 2.8 we will start by writing every element $P(x) \in \mathcal{Q I}_{m}\left[X_{3}\right]$ in the form

$$
P(x)=A_{000}+A_{010} x_{2}+A_{001} x_{3}+A_{011} x_{2} x_{3}+A_{002} x_{2}^{2}+A_{012} x_{2} x_{3}^{2} .
$$

Our goal is to see what conditions the coefficients $A_{i j k}$ must satisfy to assure that $P(x) \in \mathcal{Q I}_{m}\left[X_{3}\right]$. The idea is to use the fact that the spaces $\mathcal{Q} \mathcal{I}_{m}\left[X_{n}\right]$ are $S_{n}$ modules to gain information about these kinds of expansions. This given, our point of departure is the following identity in the algebra of $S_{3}$.

$$
i d=\mathcal{S}_{3}+\frac{1}{3}\left(1-s_{12}\right)\left(1+s_{23}\right)+\frac{1}{3}\left(1-s_{23}\right)\left(1+s_{12}\right)+\mathcal{A}_{3}
$$

where

$$
\mathcal{S}_{3}=\frac{1}{6}\left(1+s_{12}+s_{13}+s_{23}+(1,2,3)+(3,2,1)\right)
$$

and

$$
\mathcal{A}_{3}=\frac{1}{6}\left(1-s_{12}-s_{13}-s_{23}+(1,2,3)+(3,2,1)\right)
$$

Note that, since the operator $\mathcal{A}_{3}$ kills all the monomials $1, x_{2}, x_{3}, x_{2} x_{3}, x_{3}^{2}$, applying it to $P$ as given by (3.1) gives

$$
\mathcal{A}_{3} P=A_{012} \Pi_{3}(x) / 6
$$

with

$$
\Pi_{n}(x)=\prod_{1 \leq i<j \leq n}\left(x_{i}-x_{j}\right)
$$


However, note that it is an immediate consequence of the definition that any alternant in $\mathcal{Q I}_{m}\left[X_{n}\right]$ must be a multiple of $\Pi(x)^{2 m+1}$ by a symmetric polynomial. This implies that the symmetric polynomial $A_{012}$ must necessarily be a multiple of $\Pi_{3}(x)^{2 m}$. Note further that a multiple of $\Pi_{3}(x)^{2 m}$ by any polynomal in $x_{1}, x_{2}, x_{3}$ lies in $\mathcal{Q I}_{m}\left[X_{3}\right]$. This given, we see that $A_{012}$ may here and after be assumed to be of the form $A_{012}=B(x) \Delta_{3}(x)^{2 m}$ with $B(x)$ an arbitrary symmetric polynomial. It is also clear that $A_{000}$ can also be arbitrarily chosen. This reduces our study to the elements of $\mathcal{Q I}_{m}\left[X_{3}\right]$ which are of the form

$$
P(x)=A_{010} x_{2}+A_{001} x_{3}+A_{011} x_{2} x_{3}+A_{002} x_{3}^{2} .
$$

When we apply the identity in (3.2) to this expansion we derive that

$$
P(x)=A(x)+\frac{1}{3}\left(1-s_{12}\right)\left(1+s_{23}\right) P(x)+\frac{1}{3}\left(1-s_{23}\right)\left(1+s_{12}\right) P(x)
$$

with $A(x)$ a suitable symmetric polynomial. This is because $\mathcal{A}_{3}$ kills every monomial in (3.3) and $\mathcal{S}_{3}$ sends every monomial into a symmetric function .

Now we see that

$$
\left(1+s_{23}\right) P(x)=A_{010}\left(x_{2}+x_{3}\right)+A_{001}\left(x_{2}+x_{3}\right)+2 A_{011} x_{2} x_{3}+A_{002}\left(x_{2}^{2}+x_{3}^{2}\right)
$$

but we can easily check that we have

$$
x_{2}^{2}+x_{3}^{2}=-x_{2} x_{3}-e_{2}+e_{1}\left(x_{2}+x_{3}\right)
$$

Using this (3.4) becomes

$$
\left(1+s_{23}\right) P(x)=-A_{002} e_{2}+\left(A_{010}+A_{001}+e_{1} A_{002}\right)\left(x_{2}+x_{3}\right)+\left(2 A_{011}-A_{002}\right) x_{2} x_{3}+
$$

Note further that

$$
\begin{aligned}
\left(1+s_{12}\right) P(x) & =A_{010}\left(x_{1}+x_{2}\right)+2 A_{001} x_{3}+A_{011}\left(x_{1}+x_{2}\right) x_{3}+2 A_{002} x_{3}^{2} \\
& =A_{010}\left(e_{1}-x_{3}\right)+2 A_{001} x_{3}+A_{011}\left(e_{1}-x_{3}\right) x_{3}+2 A_{002} x_{3}^{2} \\
& =A_{010} e_{1}+\left(2 A_{001}-A_{010}+e_{1} A_{011}\right) x_{3}+\left(2 A_{002}-A_{011}\right) x_{3}^{2}
\end{aligned}
$$

This reduces our study to elements of $\mathcal{Q} \mathcal{I}_{m}\left[X_{3}\right]$ of the form

$$
P_{1}(x)=A_{1}\left(x_{2}+x_{3}\right)+B_{1} x_{2} x_{3}
$$

and elements of the form

$$
P_{2}(x)=A_{2} x_{3}+B_{2} x_{3}^{2}
$$

together with their images $s_{12} P_{1}$ and $s_{23} P_{2}$.

Now it develops that we have the following remarkably simple criterion.

Theorem 3.6 The polynomials $P_{1}=A_{1}\left(x_{2}+x_{3}\right)+B_{1} x_{2} x_{3}$ and $P_{2}=A_{2} x_{3}+B_{2} x_{3}^{2}$, with $A_{1}, A_{2}, B_{1}, B_{2}$ symmetric, are m-quasi-invariant if and if only we have

$$
\begin{array}{ll}
\text { a) } A_{1}=-\delta_{12} x_{1}\left(x_{1}-x_{3}\right)^{2 m} \theta_{1}(x) & B_{1}=\delta_{12}\left(x_{1}-x_{3}\right)^{2 m} \theta_{1}(x) \\
\text { b) } A_{2}=\delta_{12}\left(x_{2}+x_{3}\right)\left(x_{1}-x_{3}\right)^{2 m} \theta_{2}(x) & B_{2}=-\delta_{12}\left(x_{1}-x_{3}\right)^{2 m} \theta_{2}(x)
\end{array}
$$

where $\theta_{1}$ and $\theta_{2}$ are any polynomials that satisfy the two conditions

$$
\text { a) } s_{13} \theta=\theta, \quad \text { b) } \delta_{23} \delta_{12}\left(x_{1}-x_{3}\right)^{2 m} \theta=0,
$$


Proof We begin by proving necessity. To this end note that for $P_{1}(x)$ to be $m$-quasiinvariant we must have

$$
\left(1-s_{13}\right) P_{1}(x)=\left(x_{1}-x_{3}\right)^{2 m+1} \theta_{1}(x)
$$

with $\theta_{1}$ a polynomial in $\mathbf{Q}\left[X_{3}\right]$ satisfying the condition

$$
s_{13} \theta_{1}=\theta_{1}
$$

In fact, applying $1+s_{13}$ to $(3.9)$ gives

$$
0=\left(x_{1}-x_{3}\right)^{2 m+1} \theta_{1}(x)-\left(x_{1}-x_{3}\right)^{2 m+1} s_{13} \theta_{1}(x)
$$

and (3.10) follows upon division by $\left(x_{1}-x_{3}\right)^{2 m+1}$. On the other hand the symmetry of $A_{1}, B_{1}$ gives

$$
\left(1-s_{13}\right) P_{1}=A_{1}\left(x_{3}-x_{1}\right)+B_{1} x_{2}\left(x_{3}-x_{1}\right)
$$

using this in (3.9) we get

$$
A_{1}\left(x_{3}-x_{1}\right)+B_{1} x_{2}\left(x_{3}-x_{1}\right)=\left(x_{1}-x_{3}\right)^{2 m+1} \theta_{1}(x)
$$

or better

$$
A_{1}+B_{1} x_{2}=-\left(x_{1}-x_{3}\right)^{2 m} \theta_{1}(x) .
$$

Using again the symmetry of $A_{1}, B_{1}$, applying $\delta_{12}$ to both sides of (3.11) we obtain

$$
-B_{1}=-\delta_{12}\left(x_{1}-x_{3}\right)^{2 m} \theta_{1}(x) .
$$

Finally, multiplying by $x_{1}$ both sides of (3.11) and applying $\delta_{12}$ gives

$$
A_{1}=-\delta_{12} x_{1}\left(x_{1}-x_{3}\right)^{2 m} \theta_{1}(x) .
$$

This proves (3.7) (a). Similarly for $P_{2}$ to be $m$-quasi-invariant we must have

$$
\left(1-s_{13}\right) P_{2}=\left(x_{1}-x_{3}\right)^{2 m+1} \theta_{2}(x)
$$

for a suitable $\theta_{2}(x)$ invariant under $s_{13}$. But the symmetry of $A_{2}, B_{2}$, gives

$$
\left(1-s_{13}\right) P_{2}=A_{2}\left(x_{3}-x_{1}\right)+B_{2}\left(x_{3}^{2}-x_{1}^{2}\right)
$$

using this in (3.14) and cancelling the common factor we get

$$
-A_{2}-B_{2}\left(x_{1}+x_{3}\right)=\left(x_{1}-x_{3}\right)^{2 m} \theta_{2}(x) .
$$

Proceeding as before, using again the symmetry of $A_{2}, B_{2}$, we obtain

$$
B_{2}=-\delta_{12}\left(x_{1}-x_{3}\right)^{2 m} \theta_{2}(x)
$$


Finally, multiplying both sides by $x_{2}+x_{3}$ and applying $\delta_{12}$ we get

$$
A_{2}=\delta_{12}\left(x_{2}+x_{3}\right)\left(x_{1}-x_{3}\right)^{2 m} \theta_{2}(x) \text {. }
$$

This proves (3.7) (b). To complete our proof of necessity, we are only left to show that $\theta_{1}$ and $\theta_{2}$ must satisfy (3.8) (b). It turns out that (3.8) (b) is all we need to assure the symmetry of $A_{1}, A_{2}, B_{1}, B_{2}$. To show this it is convenient to set

$$
H_{i}=-\left(x_{1}-x_{3}\right)^{2 m} \theta_{i}(x)
$$

so that (3.12), (3.13), (3.18) and (3.19) become

$$
\begin{array}{ll}
A_{1}=\delta_{12} x_{1} H_{1}, & A_{2}=-\delta_{12}\left(x_{2}+x_{3}\right) H_{2}, \\
B_{1}=-\delta_{12} H_{1} . & B_{2}=\delta_{12} H_{2} .
\end{array}
$$

Clearly, $A_{1}, A_{2}, B_{1}, B_{2}$ are symmetric if and only if they are invariant under the action of $s_{12}$ and $s_{23}$. However, since all of them are images of $\delta_{12}$ there are automatically $s_{12^{-}}$ invariant. Thus we only need to assure that they are also $s_{23}$-invariant. Note that since, when $\theta_{2}=\theta_{1}$

$$
B_{2}=-B_{1} \quad \text { and } \quad A_{2}=-\delta_{12}\left(e_{1}-x_{1}\right) H=e_{1} B_{1}+A_{1}
$$

we need only assure the $s_{23}$-invariance of $A_{1}$ and $B_{1}$. This is equivalent to the two equations

$$
\begin{aligned}
& \text { a) } \delta_{23} \delta_{12} x_{1} H_{1}=0 \\
& \text { b) } \delta_{23} \delta_{12} H_{1}=0 .
\end{aligned}
$$

It develops that the first equation here is a consequence of the second. To see this note that since (3.21) (b) implies that $\delta_{12} H_{1}$ is symmetric in particular it is left unchanged by $s_{13}$. Thus

$$
\begin{aligned}
\delta_{12} H_{1} & =s_{13} \delta_{12} H_{1} \\
(\text { by }(3.10) \text { and }(3.18)) & =s_{13} \delta_{12} s_{13} H_{1} \\
& =\delta_{32} H_{1}=-\delta_{23} H_{1} .
\end{aligned}
$$

On the other hand we see that we have (by the Leibnitz formula)

$$
\delta_{23} \delta_{12} x_{1} H_{1}=\delta_{23}\left(H_{1}+x_{2} \delta_{12} H_{1}\right)=\delta_{23} H_{1}+\delta_{12} H_{1}+x_{3} \delta_{23} \delta_{12} H_{1} .
$$

Thus (3.21) (b) implies (3.21) (a) as asserted. Recalling the definition of $H_{1}$ in (3.18), we see that the equations in $(3.21)$ reduce to

$$
\delta_{23} \delta_{12}\left(x_{1}-x_{3}\right)^{2 m} \theta_{1}=0 .
$$

This completes the proof of necessity. 
To prove sufficiency, note that the formulas in (3.7) together with the conditions in (3.8) assure that $A_{1}, A_{2}, B_{1}, B_{2}$ are symmetric, and that $P_{1}=A_{1}\left(x_{2}+x_{3}\right)+B_{1} x_{2} x_{3}$ and $P_{2}=A_{2} x_{3}+B_{2} x_{3}^{2}$ satisfy
a) $\left(1-s_{13}\right) P_{1}=\left(x_{1}-x_{3}\right)^{2 m+1} \theta_{1}$
b) $\left(1-s_{13}\right) P_{2}=\left(x_{1}-x_{3}\right)^{2 m+1} \theta_{2}$

Indeed, from (3.7) we derive that

$$
\begin{aligned}
\delta_{13} P_{1} & =-A_{1}-B_{1} x_{2} \\
& =\frac{1}{x_{1}-x_{2}}\left(\left(1-s_{12}\right)\left(x_{1}\left(x_{1}-x_{3}\right)^{2 m} \theta_{1}(x)\right)-\left(\left(1-s_{12}\right)\left(x_{1}-x_{3}\right)^{2 m} \theta_{1}(x)\right) x_{2}\right) \\
& =\left(x_{1}-x_{3}\right)^{2 m} \theta_{1}(x)+\frac{1}{x_{1}-x_{2}}\left(-s_{12}\left(x_{1}\left(x_{1}-x_{3}\right)^{2 m} \theta_{1}(x)\right)+s_{12}\left(x_{1}\left(x_{1}-x_{3}\right)^{2 m} \theta_{1}(x)\right)\right)
\end{aligned}
$$

and this just another way of writing (3.21) (a). An entirely analogous calculation gives (3.21) (b).

Now the invariance of $A_{1}, A_{2}, B_{1}, B_{2}$ assures that

$$
\text { a) }\left(1-s_{23}\right) P_{1}=0 \quad \text { b) }\left(1-s_{12}\right) P_{2}=0
$$

On the other hand hitting (3.22) (a) by $s_{23}$ and (3.22) (b) by $s_{12}$ we get
a) $\left(1-s_{12}\right) s_{23} P_{1}=\left(x_{1}-x_{2}\right)^{2 m+1} s_{23} \theta_{1}$
b) $\left(1-s_{23}\right) s_{12} P_{2}=\left(x_{2}-x_{3}\right)^{2 m+1} s_{12} \theta_{2}$

and from (3.23) we finally derive that
a) $\left(1-s_{12}\right) P_{1}=\left(x_{1}-x_{2}\right)^{2 m+1} s_{23} \theta_{1}$
b) $\left(1-s_{23}\right) P_{2}=\left(x_{2}-x_{3}\right)^{2 m+1} s_{12} \theta_{2}$

Thus the $m$-quasi-invariance of $P_{1}$ and $P_{2}$ is assured and our proof is complete.

Our next task is to find all solutions $\theta$ of the system

$$
\begin{aligned}
& \text { a) } \delta_{23} \delta_{12}\left(x_{1}-x_{3}\right)^{2 m} \theta=0, \\
& \text { b) } s_{13} \theta=\theta .
\end{aligned}
$$

To work with expansions in the Artin basis $\mathcal{A R T}(3)$ it will be more convenient to solve the system

$$
\begin{aligned}
& \text { a) } \delta_{13} \delta_{12}\left(x_{2}-x_{3}\right)^{2 m} \theta=0, \\
& \text { b) } s_{23} \theta=\theta .
\end{aligned}
$$

There is no loss here since applying the transposition $s_{12}$ to (3.24) gives

$$
\begin{aligned}
& \text { a) } \delta_{13} \delta_{12}\left(x_{2}-x_{3}\right)^{2 m} s_{12} \theta=0, \\
& \text { b) } s_{23} s_{12} \theta=s_{12} \theta .
\end{aligned}
$$

So if $\theta$ satifies (3.24) then $s_{12} \theta$ satisfies (3.25) and vice versa.

Now note that expanding $\left(x_{2}-x_{3}\right)^{2 m}$ in terms of $\mathcal{A R T}(3)$ we obtain that

$$
\left(x_{2}-x_{3}\right)^{2 m}=A_{m}+B_{m}\left(x_{2}+x_{3}\right)+C_{m} x_{2} x_{3} .
$$


with $A_{m}, B_{m}, C_{m}$ suitable symmetric polynomials of degrees $2 m 2 m-12 m-2$ respectively. For the same reason any solution $\theta$ of (3.25) (b) must have the form

$$
\theta=a+b\left(x_{2}+x_{3}\right)+c x_{2} x_{3} .
$$

Our task then is to find all triplets $(a, b, c)$ such that the resulting $\theta$ satisfies also (3.25) (a). To carry this out we need the following auxiliary fact.

Proposition 3.26 Let

$$
H_{i}=A_{i}+B_{i}\left(x_{2}+x_{3}\right)+C_{i} x_{2} x_{3} \quad(\text { with } i=1,2)
$$

then we shall have

$$
\delta_{13} \delta_{12} H_{1} H_{2}=0
$$

if and only if

$$
C_{1}\left(A_{2}+B_{2} e_{1}+C_{2} e_{2}\right)+B_{1}\left(B_{2}+C_{2} e_{1}\right)+A_{1} C_{2}=0 .
$$

Proof Note that we have

$$
\begin{aligned}
\delta_{13} \delta_{12} H_{1} H_{2}= & \delta_{13}\left(\left(\delta_{12} H_{1}\right) H_{2}+\left(s_{12} H_{1}\right) \delta_{12} H_{2}\right) \\
= & \left(\delta_{13} \delta_{12} H_{1}\right) H_{2}+\left(s_{13} \delta_{12} H_{1}\right) \delta_{13} H_{2} \\
& \quad+\left(\delta_{13} s_{12} H_{1}\right) \delta_{12} H_{2}+\left(s_{13} s_{12} H_{1}\right) \delta_{13} \delta_{12} H_{2}
\end{aligned}
$$

Now using the expressions in (3.28) we derive that

$$
\begin{aligned}
\delta_{12} H_{i} & =-B_{i}-C_{i} x_{3} \\
s_{13} \delta_{12} H_{i} & =-B_{i}-C_{i} x_{1} \\
\delta_{13} H_{i} & =-B_{i}-C_{i} x_{2} \\
\delta_{13} \delta_{12} H_{i} & =C_{i} \\
s_{13} H_{i} & =A_{i}+B_{i}\left(x_{1}+x_{2}\right)+C_{i} x_{1} x_{2} \\
s_{12} H_{i} & =A_{i}+B_{i}\left(x_{1}+x_{3}\right)+C_{i} x_{1} x_{3} \\
s_{13} s_{12} H_{i} & =A_{i}+B_{i}\left(x_{1}+x_{3}\right)+C_{i} x_{1} x_{3} \\
\delta_{13} s_{12} H_{i} & =0
\end{aligned}
$$

This reduces (3.31) to

$$
\begin{aligned}
\delta_{13} \delta_{12} H_{1} H_{2}=C_{1}\left(A_{2}\right. & \left.+B_{2}\left(x_{2}+x_{3}\right)+C_{2} x_{2} x_{3}\right)+\left(-B_{1}-C_{1} x_{1}\right)\left(-B_{2}-C_{2} x_{2}\right) \\
& +\left(A_{1}+B_{1}\left(x_{1}+x_{3}\right)+C_{1} x_{1} x_{3}\right) C_{2}
\end{aligned}
$$

Clearly this expression vanishes identically if and only if

$$
C_{1} A_{2}+B_{1} B_{2}+A_{1} C_{2}+C_{1} B_{2} e_{1}+B_{1} C_{2} e_{1}+C_{1} C_{2} e_{2}=0 .
$$

Grouping terms according to $C_{1}, B_{1}, A_{1}$ yields (3.29) precisely as asserted.

This immediately brings us to the next step in our development. 
Theorem 3.30 Recalling that

$$
\left(x_{2}-x_{3}\right)^{2 m}=A_{m}+B_{m}\left(x_{2}+x_{3}\right)+C_{m} x_{2} x_{3} \quad \text { and } \quad \theta=a+b\left(x_{2}+x_{3}\right)+c x_{2} x_{3},
$$

with $A_{m}, B_{m}, C_{m}, a, b, c$ suitable symmetric polynomials. We shall have

$$
\delta_{13} \delta_{12}\left(x_{2}-x_{3}\right)^{2 m} \theta=0
$$

if and only if

$$
c \bar{A}_{m}+b \bar{B}_{m}+a \bar{C}_{m}=0
$$

where we have set

$$
\bar{A}_{m}=A_{m}+B_{m} e_{1}+C_{m} e_{2}, \quad \bar{B}_{m}=B_{m}+C_{m} e_{1}, \quad \bar{C}_{m}=C_{m}
$$

Proof Using (3.29) with $H_{1}=\theta$ and $H_{2}=\left(x_{2}-x_{3}\right)^{2 m}$, we get that (3.32) holds true if and only if the vector $(a, b, c)$ satisfies the equation

$$
c\left(A_{m}+B_{m} e_{1}+C_{m} e_{2}\right)+b\left(B_{m}+C_{m} e_{1}\right)+a C_{m}=0,
$$

and this is (3.33).

Our next task is to characterize the triplets of symmetric functions $(a, b, c)$ that satisfy the equation in (3.33). This will be carried out in the next section.

\section{Some Cohen-Macaulay modules of triplets.}

To proceed we need some notation. To begin let

$$
\mathbf{R}_{x}=\mathbb{Q}\left[x_{1}, x_{2}, x_{3}\right], \quad \mathbf{R}_{e}=\mathbb{Q}\left[e_{1}, e_{2}, e_{3}\right]
$$

and note that the corresponding Hilbert series are

$$
F_{\mathbf{R}_{x}}(t)=\frac{1}{(1-t)^{3}} \quad F_{\mathbf{R}_{x}}(t)=\frac{1}{(1-t)\left(1-t^{2}\right)\left(1-t^{3}\right)} .
$$

Now set

$$
\mathbf{R}_{x}^{3}=\left\{(a, b, c): a, b, c \in \mathbf{R}_{x}\right\}, \quad \mathbf{R}_{e}^{3}=\left\{(a, b, c): a, b, c \in \mathbf{R}_{e}\right\}
$$

and let us make $\mathbf{R}_{x}^{3}$ and $\mathbf{R}_{e}^{3}$ into graded modules by saying that a triplet $(a, b, c)$ is "homogeneous of degree $k$ " if and only $a, b, c$ are homogeneous of degrees $k, k-1, k-2$ respectively. We shall view the solution spaces

$$
\begin{aligned}
& \mathcal{M}_{m}(x)=\left\{(a, b, c) \in \mathbf{R}_{x}: c \bar{A}_{m}+b \bar{B}_{m}+a \bar{C}_{m}=0\right\} \\
& \mathcal{M}_{m}(e)=\left\{(a, b, c) \in \mathbf{R}_{x}: c \bar{A}_{m}+b \bar{B}_{m}+a \bar{C}_{m}=0\right\}
\end{aligned}
$$

as a graded submodules of $\mathbf{R}_{x}^{3}$ and $\mathbf{R}_{e}^{3}$ respectively. Now we have the following crucial Hilbert series identities 


\section{Proposition 4.2}

$$
\begin{aligned}
& \text { 1) } F_{\mathcal{M}_{m}(x)}(t)=(1+t)\left(1+t+t^{2}\right) F_{\mathcal{M}_{m}(e)}(t) \\
& \text { 2) } t^{2 m-2} F_{\mathcal{M}_{m}(x)}(t)=F_{\left(A_{m}, B_{m}, C_{m}\right)_{\mathbf{R}_{x}}}(t)+\frac{t^{2 m-2}+t^{2 m-1}+t^{2 m}-1}{(1-t)^{3}}
\end{aligned}
$$

Proof Note that by definition $(u, v, w) \in \mathcal{M}_{m}(x)$ if and only if

$$
w \bar{A}_{m}+v \bar{B}_{m}+u \bar{C}_{m}=0 .
$$

Now Theorem 2.8 gives the expansions

$$
u=\sum_{x^{\epsilon} \in \mathcal{A} \mathcal{R} \mathcal{T}(3)} x^{\epsilon} a_{\epsilon}, \quad v=\sum_{x^{\epsilon} \in \mathcal{A R} \mathcal{T}(3)} x^{\epsilon} b_{\epsilon}, \quad w=\sum_{x^{\epsilon} \in \mathcal{A} \mathcal{R} \mathcal{T}(3)} x^{\epsilon} c_{\epsilon},
$$

using this in (4.4) gives

$$
\sum_{x^{\epsilon} \in \mathcal{A} \mathcal{R} \mathcal{T}(3)} x^{\epsilon}\left(c_{\epsilon} \bar{A}_{m}+b_{\epsilon} \bar{B}_{m}+a_{\epsilon} \bar{C}_{m}\right)=0
$$

and the uniqueness part of Theorem 2.8 now yields that for all $x^{\epsilon} \in \mathcal{A} \mathcal{R} \mathcal{T}$ (3) we must have

$$
c_{\epsilon} \bar{A}_{m}+b_{\epsilon} \bar{B}_{m}+a_{\epsilon} \bar{C}_{m}=0 .
$$

In other words, each triplet $(u, v, w) \in \mathcal{M}_{m}(x)$ decomposes into a linear combination of triplets $\left(a_{\epsilon}, b_{\epsilon}, c_{\epsilon}\right) \in \mathcal{M}_{m}(e)$ with coefficients $x^{\epsilon} \in \mathcal{A R} \mathcal{T}(3)$. But then again the uniqueness part of Theorem 2.8 forces this decomposition to be unique. In symbols we may write

$$
\mathcal{M}_{m}(x)=\bigoplus_{x^{\epsilon} \in \mathcal{A R T}(3)} x^{\epsilon} \mathcal{M}_{m}(e)
$$

This implies the Hilbert series identity

$$
F_{\mathcal{M}_{m}(x)}(t)=\sum_{x^{\epsilon} \in \mathcal{A} \mathcal{R} \mathcal{T}(3)} t^{\text {degree }\left(x^{\epsilon}\right)} F_{\mathcal{M}_{m}(e)}(t)
$$

and (4.3) (1) follows since

$$
\sum_{x^{\epsilon} \in \mathcal{A R T}(3)} t^{\text {degree }\left(x^{\epsilon}\right)}=(1+t)\left(1+t+t^{2}\right) .
$$

To prove (4.3) (2) it is convenient to set

$$
\mathcal{W}_{m}(x)=\mathbf{R}_{x} /\left(A_{m}, B_{m}, C_{m}\right)_{\mathbf{R}_{x}}
$$

We clearly see from (3.33) that

$$
\left(\bar{A}_{m}, \bar{B}_{m}, \bar{B}_{m}\right)_{\mathbf{R}_{x}}=\left(A_{m}, B_{m}, C_{m}\right)_{\mathbf{R}_{x}}
$$


SO

$$
\mathcal{W}_{m}(x)=\mathbf{R}_{x} /\left(\bar{A}_{m}, \bar{B}_{m}, \bar{C}_{m}\right)_{\mathbf{R}_{x}} .
$$

Clearly $F_{\mathcal{W}_{m}(x)}(t)$ is given by the difference between the Hilbert series of $\mathbf{R}_{x}$ and the Hilbert series of the ideal $\left(\bar{A}_{m}, \bar{B}_{m}, \bar{C}_{m}\right)_{\mathbf{R}_{x}}$. In symbols

$$
F_{W_{m}(x)}(t)=\frac{1}{(1-t)^{3}}-F_{\left(\bar{A}_{m}, \bar{B}_{m}, \bar{C}_{m}\right)}(t)
$$

Now, by definition

$$
\left(\bar{A}_{m}, \bar{B}_{m}, \bar{C}_{m}\right)_{\mathbf{R}_{x}}=\left\{c \bar{A}_{m}+b \bar{B}_{m}+a \bar{C}_{m}: a, b, c \in \mathbf{R}_{x}\right\} .
$$

Note further that the polynomial $c \bar{A}_{m}+b \bar{B}_{m}+a \bar{C}_{m}$ is homogeneous of degree $k+2 m-2$ if and only if $a, b, c$ are respectively homogeneous of degrees $k, k-1, k-2$. Clearly the dimension of the space of such triplets $a, b, c$ is given by the expression

$$
\left.F_{\mathbf{R}_{x}}(t)\right|_{t^{k}}+\left.F_{\mathbf{R}_{x}}(t)\right|_{t^{k-1}}+\left.F_{\mathbf{R}_{x}}(t)\right|_{t^{k-2}} .
$$

To get the dimension of the degree $k+2 m-2$ homogeneous component of the ideal $\left(\bar{A}_{m}, \bar{B}_{m}, \bar{C}_{m}\right)_{\mathbf{R}_{x}}$ we must subtract from (4.7) the dimension of the collection of all triplets $(a, b, c) \in \mathcal{M}_{m}(x)$ which are homogeneous of degree $k$. This may be written as

$$
\left.F_{\mathbf{R}_{x}}(t)\right|_{t^{k}}+\left.F_{\mathbf{R}_{x}}(t)\right|_{t^{k-1}}+\left.F_{\mathbf{R}_{x}}(t)\right|_{t^{k-2}}-\left.F_{\mathcal{M}_{m}(x)}(t)\right|_{t^{k}} .
$$

Multiplying by $t^{2 m-2+k}$ and summing for $k \geq 0$ gives the identity

$$
\begin{aligned}
F_{\left(\bar{A}_{m}, \bar{B}_{m}, \bar{C}_{m} \mathbf{R}_{x}\right.}(t)=t^{2 m-2} & \left.\sum_{k \geq 0} F_{\mathbf{R}_{x}}(t)\right|_{t^{k}} t^{k} \\
& +\left.t^{2 m-1} \sum_{k \geq 1} F_{\mathbf{R}_{x}}(t)\right|_{t^{k-1}} t^{k-1} \\
& +\left.t^{2 m} \sum_{k \geq 2} F_{\mathbf{R}_{x}}(t)\right|_{t^{k-2}} t^{k-2}-t^{2 m-2} F_{\mathcal{M}_{m}(x)}(t)
\end{aligned}
$$

Now using (4.1) we derive that

$$
\begin{array}{r}
\left.t^{2 m-2} \sum_{k \geq 0} F_{\mathbf{R}_{x}}(t)\right|_{t^{k}} t^{k}+\left.t^{2 m-1} \sum_{k \geq 1} F_{\mathbf{R}_{x}}(t)\right|_{t^{k-1}} t^{k-1}+\left.t^{2 m} \sum_{k \geq 2} F_{\mathbf{R}_{x}}(t)\right|_{t^{k-2}} t^{k-2} \\
=\frac{t^{2 m-2}+t^{2 m-1}+t^{2 m}}{(1-t)^{3}}
\end{array}
$$

and (4.8) becomes

$$
F_{\left(\bar{A}_{m}, \bar{B}_{m}, \bar{C}_{m}\right)_{\mathbf{R}_{x}}}(t)=\frac{t^{2 m-2}+t^{2 m-1}+t^{2 m}}{(1-t)^{3}}-t^{2 m-2} F_{\mathcal{M}_{m}(x)}(t)
$$

Substituting this in (4.6) we finally obtain

$$
F_{W_{m}(x)}(t)=\frac{1}{(1-t)^{3}}-\frac{t^{2 m-2}+t^{2 m-1}+t^{2 m}}{(1-t)^{3}}+t^{2 m-2} F_{\mathcal{M}_{m}(x)}(t) .
$$


and this is simply another way of writing (4.3) (2). Our proof is thus complete.

We shall show in the next section that

$$
F_{\mathcal{W}_{m}(x)}(t)=\frac{\left(1+t^{m}+t^{m-1}\right)\left(1-t^{m}\right)\left(1-t^{m-1}\right)}{(1-t)^{3}}
$$

and we can state

Theorem 4.11 Upon the validity of (4.10) it follows that

$$
F_{\mathcal{M}_{m}(e)}(t)=\frac{t^{m}+t^{m+1}}{(1-t)\left(1-t^{2}\right)\left(1-t^{3}\right)}
$$

Proof Using (4.10) in (4.3) (2) gives

$$
\begin{aligned}
t^{2 m-2} F_{\mathcal{M}_{m}(x)}(t) & =\frac{\left(1+t^{m}+t^{m-1}\right)\left(1-t^{m}\right)\left(1-t^{m-1}\right)}{(1-t)^{3}}+\frac{t^{2 m-2}+t^{2 m-1}+t^{2 m}-1}{(1-t)^{3}} . \\
& =\frac{\left(1+t^{m}+t^{m-1}\right)\left(1-t^{m}-t^{m-1}+t^{2 m-1}\right)+t^{2 m-2}+t^{2 m-1}+t^{2 m}-1}{(1-t)^{3}} . \\
& =\frac{t^{3 m-1}+t^{3 m-2}}{(1-t)^{3}} .
\end{aligned}
$$

Now from (4.3) (1) we get

$$
t^{2 m-2}(1+t)\left(1+t+t^{2}\right) F_{\mathcal{M}_{m}(e)}(t)=\frac{t^{3 m-1}+t^{3 m-2}}{(1-t)^{3}} .
$$

and (4.12) follows by cancelling the factor $t^{2 m-2}$ and division of both sides by $(1+t)(1+$ $\left.t+t^{2}\right)$.

This brings us to the crucial result of this section,

Theorem 4.13 Upon the validity of (4.10) it follows that the collection

$$
\mathcal{M}_{m}(e)=\left\{(a, b, c): c \bar{A}_{m}+b \bar{B}_{m}+a \bar{C}_{m}=0\right\}
$$

is a free $\mathbb{Q}\left[e_{1}, e_{2}, e_{3}\right]$-module of rank 2

Proof From the Hilbert series in (4.12) it follows that the subspaces $\mathcal{H}_{m}\left(\mathcal{M}_{m}(e)\right)$ and $\mathcal{H}_{m+1}\left(\mathcal{M}_{m}(e)\right)$ of homogeneous triplets of $\mathcal{M}_{m}(e)$ of degrees $m$ and $m+1$ have dimensions 1 and 2 respectively. This given, let $\Theta_{1}=\left(a_{1}, b_{1}, c_{1}\right)$ be a non trivial element of $\mathcal{H}_{m}\left(\mathcal{M}_{m}(e)\right)$ and note that $e_{1} \Theta_{1}=\left(e_{1} a_{1}, e_{1} b_{1}, e_{1} c_{1}\right) \in \mathcal{H}_{m+1}\left(\mathcal{M}_{m}(e)\right)$. This accounts for one of the 2 dimensions of $\mathcal{H}_{m+1}\left(\mathcal{M}_{m}(e)\right)$. Let us pick $\Theta_{2}=\left(a_{2}, b_{2}, c_{2}\right) \in \mathcal{H}_{m+1}\left(\mathcal{M}_{m}(e)\right)$ so that together with $e_{1} \Theta_{1}$ we have a basis for $\mathcal{H}_{m+1}\left(\mathcal{M}_{m}(e)\right)$. Now suppose that for two symmetric functions $D_{1}$ and $D_{2}$ we have

$$
D_{1} \Theta_{1}+D_{2} \Theta_{2}=0
$$


Clearly there is no loss in assuming that $D_{1}$ and $D_{2}$ have no common factor. Then it follows from (4.14) that $D_{2}$ must divide $a_{1}, b_{1}$ and $c_{1}$. But since $\Theta_{1}$ is an element of least degree in $\mathcal{M}_{m}(e)$ it follows that $D_{2}$ must be a constant and there is no loss in taking it to be -1 . So (4.14) becomes

$$
D_{1} \Theta_{1}=\Theta_{2}
$$

This given, equating the first components in (4.14) gives $D_{1} a_{1}=a_{2}$ this forces $D_{1}$ to be homogeneous of degree 1 since it is symmetric it can only be a constant multiple of $e_{1}$, but then (4.15) contraddicts our initial choice of $\Theta_{2}$. Thus there is no relation such as in (4.14). This means that the collection

$$
\left\{\Theta_{i} e_{1}^{p_{1}} e_{2}^{p_{2}} e_{3}^{p_{3}}\right\}_{\substack{i=1,2 \\ p_{i} \geq 0}}
$$

is an independent subset of $\mathcal{M}_{m}(e)$ and since it has the correct number of elements in each degree it must be a basis. In summary, $\Theta_{1}, \Theta_{2}$ generate $\mathcal{M}_{m}(e)$ as a free $\mathbb{Q}\left[e_{1}, e_{2}, e_{3}\right]$ module and our proof is complete.

We can now obtain our desired result on $S_{3} m$-quasi-invariants:

Theorem 4.16 Let $\left(a_{1}, b_{1}, c_{1}\right)$ and $\left(a_{2}, b_{2}, c_{2}\right)$ be the generators of $\mathcal{M}_{m}(e)$ of degrees $m$ and $m+1$ and set

$$
\theta_{1}=a_{1}+b_{1}\left(x_{1}+x_{3}\right)+c_{1} x_{1} x_{3}, \quad \theta_{2}=a_{2}+b_{2}\left(x_{1}+x_{3}\right)+c_{2} x_{1} x_{3} .
$$

Then $\mathcal{Q I}_{m}\left[X_{3}\right]$ is a free $\mathbf{Q}\left[e_{1}, e_{2}, e_{3}\right]$ - module with basis

$$
1, G_{1}, s_{12} G_{1}, G_{2}, s_{12} G_{2}, x_{2} x_{3}^{2} \Pi(x)^{2 m}
$$

where $\Pi(x)$ denotes the Vandermonde determinant in $x_{1}, x_{2}, x_{3}$,

$$
G_{1}=-\left(\delta_{12} x_{1}\left(x_{1}-x_{3}\right)^{2 m} \theta_{1}\right)\left(x_{2}+x_{3}\right)+\left(\delta_{12}\left(x_{1}-x_{3}\right)^{2 m} \theta_{1}\right) x_{2} x_{3}
$$

and

$$
G_{2}=-\left(\delta_{12} x_{1}\left(x_{1}-x_{3}\right)^{2 m} \theta_{2}\right)\left(x_{2}+x_{3}\right)+\left(\delta_{12}\left(x_{1}-x_{3}\right)^{2 m} \theta_{2}\right) x_{2} x_{3}
$$

Proof We have seen in section 2 that every $m$-quasi-invariant in $x_{1}, x_{2}, x_{3}$ is a sum of terms involving

(1) An invariant

(2) The polynomial $x_{2} x_{3}^{2} \Pi(x)^{2 m}$ times an invariant.

(3) An $m$-quasi-invariant $P_{1}=A_{1}\left(x_{2}+x_{3}\right)+B_{1} x_{2} x_{3}$ and its image by $s_{12}$.

(4) An $m$-quasi-invariant $P_{2}=A_{2} x_{3}^{2}+B_{2} x_{3}^{2}$ and its image by $s_{23}$. 
with $A_{1}, B_{1}, A_{2}, B_{2}$ invariants. We have also shown that for $P_{1}$ and $P_{2}$ to be $m$-quasiinvariant it is necessary and sufficient that $A_{1}, B_{1}, A_{2}, B_{2}$ have the expressions given in

(3.7). Now it develops that an $m$-quasi-invariant of the form $P_{2}=A_{2} x_{3}^{2}+B_{2} x_{3}^{2}$ can be obtained (modulo the ideal generated by $e_{1}, e_{2}, e_{3}$ ) as an $S_{3}$ image of an $m$-quasi-invariant polynomial of the form $P_{1}=A_{1}\left(x_{2}+x_{3}\right)+B_{1} x_{2} x_{3}$ In fact, note that from (3.7) (a) and (b) we derive that when $\theta_{1}=\theta_{2}=\theta$

$$
\begin{aligned}
A_{2} & =\delta_{12}\left(x_{2}+x_{3}\right)\left(x_{1}-x_{3}\right)^{2 m} \theta(x) \\
& =\delta_{12}\left(e_{1}-x_{1}\right)\left(x_{1}-x_{3}\right)^{2 m} \theta(x) \text { and } B_{2}=-B_{1} \\
& =A_{1}+e_{1} B_{1}
\end{aligned}
$$

This gives

$$
\begin{aligned}
\left(1+s_{12}\right) P_{1} & =A_{1}\left(x_{1}+x_{2}+2 x_{3}\right)+B_{1}\left(x_{1} x_{3}+x_{2} x_{3}\right) \\
& =A_{1}\left(e_{1}+x_{3}\right)+B_{1}\left(e_{1}-x_{3}\right) x_{3} \\
& =A_{1} e_{1}+\left(A_{1}+B_{1} e_{1}\right) x_{3}+B_{2} x_{3}^{2}=A_{1} e_{1}+P_{2} .
\end{aligned}
$$

Combining this with Theorem 4.11 we derive that every $m$-quasi-invariant $P$ is of the form

$$
P=U+G+s_{12} G+V x_{2} x_{3}^{2} \Pi(x)^{2 m}
$$

where $U, V$ are arbitrary invariants and

$$
G=\left(-\delta_{12} x_{1}\left(x_{1}-x_{3}\right)^{2 m} \theta(x)\right)\left(x_{2}+x_{3}\right)+\left(\delta_{12}\left(x_{1}-x_{3}\right)^{2 m} \theta(x)\right) x_{2} x_{3}
$$

where

$$
\theta=a+b\left(x_{1}+x_{3}\right)+c x_{1} x_{3} \quad \text { with }(a, b, c) \in \mathcal{M}_{m}(e)
$$

This implies that the polynomials in (4.17) span $\mathcal{Q} \mathcal{I}_{m}\left[X_{3}\right]$ as a $\mathbb{Q}\left[e_{1}, e_{2}, e_{3}\right]$-module. Since they are altogether $6=3$ ! in total, we can use Theorem 2.14 and obtain the polynomials in (4.17) are in fact a free $\mathbb{Q}\left[e_{1}, e_{2}, e_{3}\right]$-module basis for $\mathcal{Q} \mathcal{I}_{m}\left[X_{3}\right]$.

\section{Determining the quotient $R_{x} /\left(A_{m}, b_{m}, C_{m}\right)_{R_{x}}$.}

Our first task is to construct the Gröbner basis of the ideal $\left(A_{m}, B_{m}, C_{m}\right)_{\mathbf{R}_{x}}$. The following identities open up a surprising path.

Proposition 5.1 Denoting by $\Pi(x)$ the vandemonde determinant in $x_{1}, x_{2}, x_{3}$ we have

$$
\begin{aligned}
& P_{m}(x)=\Pi(x) A_{m}(x)=x_{3}^{2}\left(x_{1}-x_{2}\right)^{2 m+1}+x_{1}^{2}\left(x_{2}-x_{3}\right)^{2 m+1}+x_{2}^{2}\left(x_{3}-x_{1}\right)^{2 m+1} \\
& Q_{m}(x)=\Pi(x) B_{m}(x)=-x_{3}\left(x_{1}-x_{2}\right)^{2 m+1}-x_{1}\left(x_{2}-x_{3}\right)^{2 m+1}-x_{2}\left(x_{3}-x_{1}\right)^{2 m+1} \\
& R_{m}(x)=\Pi(x) C_{m}(x)=\left(x_{1}-x_{2}\right)^{2 m+1}+\left(x_{2}-x_{3}\right)^{2 m+1}+\left(x_{3}-x_{1}\right)^{2 m+1}
\end{aligned}
$$


Proof Because of uniqueness of the expansions in terms of $\mathcal{A R T}(3)$ and the symmetry of $A_{m}, b_{m}, C_{m}$ it is sufficient to verify the identity in (3.27). In other words we need to show that

$$
\Pi(x)\left(x_{2}-x_{3}\right)^{2 m}=P_{m}+Q_{m}\left(x_{2}+x_{3}\right)+R_{m} x_{2} x_{3},
$$

Now denoting by $R H S$ the right hand side and using (5.2) we get

$$
\begin{aligned}
\text { RHS }= & x_{3}^{2}\left(x_{1}-x_{2}\right)^{2 m+1}+x_{1}^{2}\left(x_{2}-x_{3}\right)^{2 m+1}+x_{2}^{2}\left(x_{3}-x_{1}\right)^{2 m+1} \\
& -\left(x_{2}+x_{3}\right)\left(x_{3}\left(x_{1}-x_{2}\right)^{2 m+1}-x_{1}\left(x_{2}-x_{3}\right)^{2 m+1}-x_{2}\left(x_{3}-x_{1}\right)^{2 m+1}\right) \\
= & x_{2} x_{3}\left(\left(x_{1}-x_{2}\right)^{2 m+1}+\left(x_{2}-x_{3}\right)^{2 m+1}+\left(x_{3}-x_{1}\right)^{2 m+1}\right) \\
= & \left(x_{1}^{2}-x_{2} x_{1}-x_{3} x_{1}+x_{2} x_{3}\right)\left(x_{2}-x_{3}\right)^{2 m+1} \\
= & \left(x_{1}-x_{2}\right)\left(x_{1}-x_{3}\right)\left(x_{3}-x_{1}\right)^{2 m+1}=\pi(x)\left(x_{3}-x_{1}\right)^{2 m} .
\end{aligned}
$$

This proves (5.3).

To proceed it will be convenient to make a change of variables and set

$$
x_{1}=y+u, \quad x_{2}=y, \quad x_{3}=y-v .
$$

This gives

$$
x_{1}-x_{2}=u, \quad x_{2}-x_{3}=v, \quad x_{3}-x_{1}=-u-v .
$$

Thus we may write

$$
\begin{aligned}
A_{m} & =\frac{(y-v)^{2} u^{2 m+1}+(y+u)^{2} v^{2 m+1}-y^{2}(u+v)^{2 m+1}}{-u v(u+v)} \\
B_{m} & =\frac{-(y-v) u^{2 m+1}-(y+u) v^{2 m+1}+y(u+v)^{2 m+1}}{-u v(u+v)} \\
C_{m} & =\frac{u^{2 m+1}+v^{2 m+1}-(u+v)^{2 m+1}}{-u v(u+v)}
\end{aligned}
$$

Now note that

$$
B_{m}=-y C_{m}+\frac{v u^{2 m+1}-u v^{2 m+1}}{-u v(u+v)}=-y C_{m}+\frac{v^{2 m}-u^{2 m}}{u+v}=-y C_{m}+\widetilde{B}_{m},
$$

where we have set

$$
\widetilde{B}_{m}=\frac{v^{2 m}-u^{2 m}}{u+v} .
$$

Similarly we get

$$
\begin{aligned}
A_{m} & =y^{2} C_{m}+\frac{-2 y v u^{2 m+1}+2 y u v^{2 m+1}}{-u v(u+v)}+\frac{v^{2} u^{2 m+1}+u^{2} v^{2 m+1}}{-u v(u+v)} \\
& =y^{2} C_{m}-2 y \frac{u^{2 m}-v^{2 m}}{-(u+v)}-\frac{v u^{2 m}+u v^{2 m}}{u+v}=y^{2} C_{m}-2 y \widetilde{B}_{m}-\widetilde{A}_{m}
\end{aligned}
$$


where we have set

$$
\widetilde{A}_{m}=\frac{v u^{2 m}+u v^{2 m}}{u+v}
$$

and we clearly see that we have

$$
\left(A_{m}, B_{m}, C_{m}\right)_{\mathbb{Q}[u, v, y]}=\left(\widetilde{A}_{m}, \widetilde{B}_{m}, C_{m}\right)_{\mathbb{Q}[u, v, y]}
$$

Note further that from (5.7) and (5.8) we get

$$
\widetilde{A}_{m}+v \widetilde{B}_{m}=\frac{v u^{2 m}+u v^{2 m}}{u+v}+v \frac{v^{2 m}-u^{2 m}}{u+v}=v^{2 m}
$$

and (5.9) can be then replaced by

$$
\left(A_{m}, B_{m}, C_{m}\right)_{\mathbb{Q}[u, v, y]}=\left(v^{2 m}, \widetilde{B}_{m}, C_{m}\right)_{\mathbb{Q}[u, v, y]} .
$$

To work with this ideal will be convenient to set, here and after

$$
P(t)=\frac{1-t^{2 m}}{1+t} \quad \text { and } \quad Q(t)=\frac{(1+t)^{2 m+1}-t^{2 m+1}-1}{t(1+t)}
$$

So that we may write

$$
\widetilde{B}_{m}(u, v)=v^{2 m-1} P(u / v) \quad \text { and } \quad C_{m}(u, v)=v^{2 m-2} Q(u / v) .
$$

We can easily see that

$$
P(t)=\sum_{r=0}^{2 m-1}(-t)^{r} \quad \text { and } \quad Q(t)=\sum_{r=1}^{2 m-1} t^{r-1} \sum_{s=1}^{r \wedge(2 m-r)}\left(\begin{array}{c}
2 m+1 \\
s
\end{array}\right)(-1)^{s-r}
$$

However these expansions will play no role. All we need to know is that $P(t)$ and $Q(t)$ are of degrees $2 m-1$ and $2 m-2$ respectively and that the following technical result holds true.

Lemma 5.13 Suppose that we have

$$
R(t)=a(t) P(t)+b(t) Q(t),
$$

with $a(t), b(t)$ polynomials of degrees bounded by some $j<m$, Then the polynomial $R(t)$ must have degree at least $2 m-2-j$

Proof Set

$$
S(t)=t^{2 m-1}-1 \quad \text { and } \quad T(t)=(1+t)^{2 m-1}-(1+t)
$$

then multiplying $(5.14)$ by $t(1+t)$ gives

$$
\begin{aligned}
t(1+t) R(t) & =a(t)\left(t-t^{2 m+1}\right)+b(t)\left((1+t)^{2 m+1}-t^{2 m+1}-1\right) \\
& =(a(t)+b(t))\left(t-t^{2 m+1}\right)+b(t)\left((1+t)^{2 m+1}-(1+t)\right) .
\end{aligned}
$$


In summary, we get that for some polynomials $c(t)$ and $b(t)$ of degrees bounded by $j$ we have

$$
t(1+t) R(t)=-c(t) S(t)+b(t) T(t),
$$

Now, proceeding by contraddiction, suppose if possible that the degree of $R(t)$ is strictly less than $2 m-2-j$. Then differentiating both sides of (5.16) $2 m-j$ times gives

$$
\left(\frac{d}{d t}\right)^{2 m-j} a(t) S(t)=\left(\frac{d}{d t}\right)^{2 m-j} b(t) T(t)
$$

or better

$$
\sum_{i=0}^{2 m-j}\left(\begin{array}{c}
2 m-j \\
i
\end{array}\right) c^{(i)}(t) S^{(2 m-j-i)}(t)=\sum_{i=0}^{2 m-j}\left(\begin{array}{c}
2 m-j \\
i
\end{array}\right) b^{(i)}(t) T^{(2 m-j-i)}(t)
$$

since $j<m$ we will have $2 m-j>m$ and since by assumption both $b$ and $c$ have degrees bounded by $j<m<2 m-j$, these sums need only be carried out for $i \leq j$, giving

$$
\sum_{i=0}^{j}\left(\begin{array}{c}
2 m-j \\
i
\end{array}\right) c^{(i)}(t) S^{(2 m-j-i)}(t)=\sum_{i=0}^{j}\left(\begin{array}{c}
2 m-j \\
i
\end{array}\right) b^{(i)}(t) T^{(2 m-j-i)}(t)
$$

This means that both $S(t)$ and $T(t)$ are differentiated at least $2 m-j>m$ times, that is at least two times, since $m \geq 1$. This means that we can ignore the linear terms and obtain

$S^{(2 m-j-i)}(t)=(2 m+1)_{2 m-j-i} t^{1+i+j} \quad$ and $\quad T^{(2 m-j-i)}(t)=(2 m+1)_{2 m-j-i}(1+t)^{1+i+j}$.

So (5.17) may be rewritten as

$$
\begin{aligned}
& t^{1+j} \sum_{i=0}^{j}\left(\begin{array}{c}
2 m-j \\
i
\end{array}\right) c^{(i)}(t)(2 m+1)_{2 m-j-i} t^{i} \\
& \quad=(1+t)^{1+j} \sum_{i=0}^{j}\left(\begin{array}{c}
2 m-j \\
i
\end{array}\right) b^{(i)}(t)(2 m+1)_{2 m-j-i}(1+t)^{i}
\end{aligned}
$$

Setting

$$
\begin{aligned}
& U(t)=\sum_{i=0}^{j}\left(\begin{array}{c}
2 m-j \\
i
\end{array}\right) c^{(i)}(t)(2 m+1)_{2 m-j-i} t^{i} \\
& \quad \text { and } \quad V(t)=\sum_{i=0}^{j}\left(\begin{array}{c}
2 m-j \\
i
\end{array}\right) b^{(i)}(t)(2 m+1)_{2 m-j-i}(1+t)^{i}
\end{aligned}
$$

from (5.18) we derive that

$$
(1+t)^{1+j} \mid U(t) \quad \text { and } \quad t^{1+j} \mid V(t)
$$

Now since both $c$ and $b$ have degrees $<j+1$, (5.18) forces both $U(t)$ and $V(t)$ to vanish. That means that we must have

$$
\left(\frac{d}{d t}\right)^{2 m-j} a(t) S(t)=0 \quad \text { and } \quad\left(\frac{d}{d t}\right)^{2 m-j} b(t) T(t)=0
$$

but that is absurd since both $a(t) S(t)$ and $b(t) T(t)$ have degrees $\geq 2 m+1$.

This brings us in a position to state and prove the crucial result of this section 
Theorem 5.19 The dlex minimal elements of the lower set of leading monomials of the ideal

$$
\left(v^{2 m}, \widetilde{B}_{m}, C_{m}\right)_{\mathbb{Q}[u, v]}
$$

with respect to the total order $u>v$ are

$$
u^{2 m-2}, v^{2} u^{2 m-3}, v^{4} u^{2 m-4}, \ldots, v^{2 i} u^{2 m-2-i} \ldots, v^{2 m-2} u^{m-1}, v^{2 m}
$$

Proof We shall start by proving that every leading monomial of the ideal in (5.20) is divisible by one of the monomials in (5.21). To begin note that if $u^{h} v^{k}$ is a monomial not divisible by any of the monomials in (5.12) then $v<2 m$ and we must have

$$
k=\left\{\begin{array}{l}
2 j \\
2 j+1
\end{array} \text { or } \quad(\text { for some } 0 \leq j<m)\right.
$$

as well as

$$
0 \leq h<2 m-2-j .
$$

Suppose if possible that a monomial $u^{h} v^{k}$ with $h, k$ satisfying (5.22) and (5.23) is the leading monomial of a homogeneous element of $M(u, v) \in\left(v^{2 m}, \widetilde{B}_{m}, C_{m}\right)_{\mathbb{Q}[u, v]}$. Then, setting

$$
r=h+k
$$

we have the expansion

$$
M(u, v)=u^{h} v^{k}+\sum_{\substack{k^{\prime}>k \\ h^{\prime}+k^{\prime}=r}} c_{h^{\prime}, k^{\prime}} u^{h^{\prime}} v^{k^{\prime}}=v^{k} R(u, v)
$$

with $R(u, v)$ homogeneous of degree $h$ and leading monomial $u^{h}$ and there will be some homogeneous polynomials $a(u, v), b(u, v), c(u, v)$ giving

$$
v^{k} R(u, v)=a(u, v) \widetilde{B}_{m}(u, v)+b(u, v) C_{m}(u, v)+c(u, v) v^{2 m}
$$

then setting $u=t v$ and denoting by $d_{a}, d_{b}$ and $d_{c}$ the degrees of $a(u, v), b(u, v)$ and $c(u, v)$ we obtain

$$
v^{k+h} R(t, 1)=v^{d_{a}+2 m-1} a(t, 1) P(t)+v^{d_{b}+2 m-2} b(t, 1) Q(t)+v^{d_{c}+2 m} c(t, 1) .
$$

This gives

$$
k+h=d_{a}+2 m-1=d_{b}+2 m-2=d_{c}+2 m,
$$

and cancelling the common factor $v^{h+k}(5.27)$ may be rewritten as

$$
R(t, 1)-c(t, 1)=a(t, 1) P(t)+b(t, 1) Q(t)
$$


Now note that from (5.22) and (5.23) we get

$$
\begin{gathered}
d_{a}=k+h-2 m+1<\left\{\begin{array}{l}
2 j+2 m-2-j-2 m+1 \quad \text { if } k=2 j \\
2 j+1+2 m-2-j-2 m+1 \text { if } k=2 j
\end{array}\right. \\
= \begin{cases}j-1 & \text { if } k=2 j \\
j & \text { if } k=2 j+1\end{cases}
\end{gathered}
$$

similarly we must also have

$$
d_{b}<\left\{\begin{array}{ll}
j \quad \text { if } k=2 j \\
j+1 \quad \text { if } k=2 j+1
\end{array} \quad \text { and } \quad d_{c}< \begin{cases}j-2 & \text { if } k=2 j \\
j-1 & \text { if } k=2 j+1\end{cases}\right.
$$

Thus in any case the polynomials $a(t, 1)$ and $b(t, 1)$ have degrees bounded by $j$. This places us in a position to use Lemma 5.13 and conclude that $R(t, 1)-c(t, 1)$ must be of degree at least $2 m-2-j$. But in any case (5.30) shows that $c(t)$ has degree at most $j-1<2 m-2-j$ thus $R(t, 1)$ itself must have degree at least $2 m-2-j$ but that contradicts (5.23). So every leading monomial of the ideal in (5.20) must be divisible by one of the monomials in (5.21) precisely as asserted.

To complete the proof we need to show that each of the monomials in (5.21) is a leading monomial. To this end we apply the Berlekamp algorithm [2] for computing the greatest common divisor of $P$ and $Q$, as given by (5.21), we obtain a sequence of polynomials

$$
Q_{i}(t) R_{i}(t) a_{i}(t) b_{i}(t) \quad(\text { for } \quad i=-1,0,1,2, \ldots, m)
$$

determined by the initial conditions

$$
\begin{array}{ccc}
R_{-1}=P & a_{-1}=1 & b_{-1}=0 \\
R_{o}=Q & a_{0}=0 & b_{0}=1
\end{array}
$$

where $Q_{i}$ and $R_{i}$ are the quotient and the remainder of the division of $R_{i-2}$ by $R_{i-1}$, in symbols

$$
R_{i-2}=R_{i-1} Q_{i}+R_{i}
$$

and $a_{i}, b_{i}$ are obtained from the recursions

$$
\text { a) } a_{i-2}=a_{i-1} Q_{i}+a_{i} \quad \text { b) } b_{i-2}=b_{i-1} Q_{i}+b_{i}
$$

This allows us to express $R_{i}$ in the form

$$
R_{i}=a_{i} P+b_{i} Q
$$

Clearly the degree of $R_{i}$ as constructed from (5.15) decreases by 1 at least at each step. Since we see from (5.14) that $R_{-1}$ has degree $2 m-1$ it follows that

$$
\operatorname{degree}\left(R_{i}\right) \leq 2 m-2-i \quad(\text { for all } i<m)
$$


Actually we will show that equality must hold here. Since as we noted this is true for $i=-1,0$, we can proceed by induction an assume that the equality

$$
\operatorname{degree}\left(R_{i}\right)=2 m-2-i
$$

has been established for all $i<j$. Now note this equality for all $i<j$ together with (5.32) gives that $Q_{i}$ is of degree 1 for all $0 \leq i \leq j$ in particular we can recursively obtain from (5.33) that $a_{j}$ and $b_{j}$ are of degrees $j-1$ and $j$ respectively. This places us in a position to apply Lemma 5.13 to (5.34) for $i=j$ that is

$$
R_{j}=a_{j} P+b_{j} Q
$$

and conclude that the degree of $R_{j}$ must be at least $2 m-2-j$ which combined with the inequality in (5.35) yields that

$$
\operatorname{degree}\left(R_{j}\right)=2 m-2-j
$$

completing the induction. Now setting $t=u / v$ in (5.35) and mutiplying both sides by $v^{2 m-2-j}$, gives

$$
v^{2 j} v^{2 m-2-j} R_{j}(u / v)=v^{j-1} a_{i}(u / v) v^{2 m-1} P(u / v)+v^{j} b_{j}(u / v) v^{2 m-2} Q(u / v),
$$

Using the relations in (5.12) this may be rewritten as

$$
v^{2 i} R(u, v)=a(u, v) \widetilde{B}_{m}(u, v)+b(u, v) C_{m}(u, v),
$$

with

$$
a(u, v)=v^{j-1} a_{j}(u / v), \quad b(u, v)=v^{j} b_{j}(u / v), \quad R(u, v)=v^{j-1} a(u / v),
$$

Now we have seen that the equality in (5.35) for all $i<m$ forces $a_{j}$ and $b_{j}$ to be of degrees $j-1$ and $j$ respectively we derive from (5.39) that $a(u, v), b(u, v)$ are homogeneous polynomials, likewise (5.37) yields that $R(u, v)$ is a homogeneous polynomial with leading monomial $u^{2 m-2-j}$. But then (5.38) proves that $v^{2 j} u^{2 m-2-j}$ is a leading monomial of the ideal $\left(v^{2 m}, \widetilde{B}_{m}, C_{m}\right) \mathbb{Q}[u, v]$. Thus our proof is complete.

It develops that Theorem 5.19 is more that is needed to establish the Hilbert series equality in (4.10). More precisely we have

Theorem 5.41 The standard basis of the quotient

$$
\mathbb{Q}[u, v] /\left(\widetilde{B}_{m}, C_{m}, v^{2 m}\right)
$$

relative to the order $u>v$ is given by the collection of monomials

$$
\begin{aligned}
& \mathcal{B}_{m}= \\
& \quad\left\{v^{2 i}, v^{2 i} u, v^{2 i} u^{2}, \ldots, v^{2 i} u^{2 m-3-i} ; v^{2 i+1}, v^{2 i+1} u, v^{2 i+1} u^{2}, \ldots, v^{2 i+1} u^{2 m-3-i}\right\}_{0 \leq i<m}
\end{aligned}
$$


In particular the Hilbert series of the quotients

$$
\mathbb{Q}[u, v, y] /\left(\widetilde{B}_{m}, C_{m}, v^{2 m}\right), \quad \mathbf{R}_{x} /\left(\bar{A}_{m}, \bar{B}_{m}, \bar{C}_{m}\right)_{\mathbf{R}_{x}}
$$

are given by the rational function

$$
\frac{\left(1-t^{m}\right)\left(1-t^{m-1}\right)\left(1+t^{m}+t^{m-1}\right)}{(1-t)^{3}}
$$

Proof Note that by Theorem 5.19, the collection in (5.40) constitutes the lower set of non-leading monomials of the ideal $\left(\widetilde{B}_{m}, C_{m}, v^{2 m}\right)$. Thus it is the standard basis as asserted. Thus the Hilbert series of the quotient $\mathbb{Q}[u, v] /\left(\widetilde{B}_{m}, C_{m}, v^{2 m}\right)$ is given by the generating function

$$
\begin{aligned}
\sum_{b \in \mathcal{B}} t^{\text {degree }(b)} & =\sum_{i=0}^{m-1}\left(t^{2 i}+t^{2 i+1}\right)\left(1+t+\cdots+t^{2 m-3-i}\right) \\
& =\frac{1+t}{1-t} \sum_{i=0}^{m-1} t^{2 i}\left(1-t^{2 m-2-i}\right) \\
& =\frac{1+t}{1-t}\left(\frac{1-t^{2 m}}{1-t^{2}}-t^{2 m-2} \frac{1-t^{m}}{1-t}\right) \\
& =\frac{\left(1-t^{m}\right)}{1-t}\left(\frac{1+t^{m}}{1-t}-\frac{t^{2 m-2}+t^{2 m-1}}{1-t}\right) \\
& =\frac{\left(1-t^{m}\right)\left(1-t^{m-1}\left(1+t^{m}+t^{m-1}\right)\right)}{(1-t)^{2}}
\end{aligned}
$$

This gives that the rational function in (5.43) gives the Hilbert series of $\mathbb{Q}[u, v, y] /$ $\left(\widetilde{B}_{m}, C_{m}, v^{2 m}\right)$. The extra factor of $(1-t)$ in the denominator accounting for the presence of the extra variable $y$. This completes our proof since the manipulations at the beginning of the section prove that the two quotients in (5.42) have the same Hilbert series.

\section{References}

[1] Yu. Berest, P. Etingof and V. Ginzburg, Cherednik algebras and differential operators on m-quasi-invariants, math. QA/011005.

[2] E. R. Berlekamp, Agebraic Coding Theory, Aegean Park Press (1984), pp. 21-30.

[3] O. A. Chalykh and A. P. Veselov, Commutative Rings of Partial Differential Operators and Lie Algebras, Comm. in Math. Phys. 126(1990), 597-611.

[4] P. Etingof and V. Ginzburg, Om m-quasi-invariants of a Coxeter group , arXiv:math.QA/0106175 v1 Jun 2001. 
[5] P. Etingof and E. Strickland, Lectures on quasi-invariants of Coxeter groups and the Cherednik algebra, arXiv:math.QA/0204104 v1 9 Apr 2002.

[6] G. Felder and A. P. Veselov, Action of Coxeter Groups on m-harmonic Polynomials and KZ equations arXiv:math.QA/0108012 v2 3 Oct 2001.

[7] M. Feigin and A. P. Veselov, Quasi-invariants of Coxeter groups and m-harmonmic polynomials, arXiv:math-ph/0105014 v1 11 May 2001.

[8] A. M. Garsia and N. Wallach, Some New Applications of Orbit Harmonics, J. of Comb. Theory Series A (to appear).

[9] A. P. Veselov, K.L. Styrkas and O. A. Chalykh, Algebraic integrability for the Schrödinger equation and finite reflection groups, (translated from Teo. i Math. Fizika, Vol 94 No 2 pp 253-275) 